\title{
Intermediate- and heavy-Higgs-boson physics at a $0.5 \mathrm{TeV} e^{+} e^{-}$collider
}

\author{
V. Barger and Kingman Cheung \\ Physics Department, University of Wisconsin, Madison, Wisconsin 53706 \\ B. A. Kniehl \\ Deutsches Elektronen-Synchrotron, D-2000 Hamburg 52, Germany \\ R. J. N. Phillips \\ Rutherford Appleton Laboratory, Chilton, Didcot, Oxon, OX11 OQX, England
}

(Received 25 February 1992)

\begin{abstract}
We explore the potential of a future $e^{+} e^{-}$collider in the $0.5 \mathrm{TeV}$ center-of-mass energy range to detect intermediate or heavy Higgs bosons in the standard model. We first briefly assess the logistics for finding a Higgs boson of intermediate mass, with $M_{Z}<m_{H}<2 M_{W}$. We then study in detail the possibility of detecting a heavy Higgs boson, with $m_{H}>2 M_{W}$, through the production of pairs of weak bosons. We quantitatively analyze the sensitivity of the process $e^{+} e^{-} \rightarrow \nu \bar{\nu} W^{+} W^{-}(Z Z)$ to the presence of a heavy-Higgs-boson resonance in the standard model. We compare this signal to various backgrounds and to the smaller signal from $e^{+} e^{-} \rightarrow Z H \rightarrow \mu^{+} \mu^{-} W^{+} W^{-}(Z Z)$, assuming the weak-boson pairs to be detected and measured in their dominant hadronic decay modes $W^{+} W^{-}(Z Z) \rightarrow 4$ jets. A related Higgs-boson signal in 6-jet final states is also estimated. We show how the main backgrounds from $e^{+} e^{-} W^{+} W^{-}(Z Z), e \nu W Z$, and $t \bar{t}$ production can be reduced by suitable acceptance cuts. Bremsstrahlung and typical beamstrahlung corrections are calculated. These corrections reduce Higgs-boson production by scattering mechanisms but increase production by annihilation mechanisms; they also smear out some dynamical features such as Jacobian peaks in $p_{T}(H)$. With all these corrections included, we conclude that it should be possible to detect a heavy-Higgs-boson signal in the $\nu \bar{\nu} W^{+} W^{-}(Z Z)$ channels up to mass $m_{H}=350 \mathrm{GeV}$.
\end{abstract}

PACS number(s): 14.80,Gt, 13.10.+q

\section{INTRODUCTION}

The structure of the electroweak symmetry-breaking mechanism is a fundamental issue in particle physics today. A promising way to probe this structure is to study the production of pairs of $W$ and $Z$ bosons at high-energy colliders, since the longitudinally polarized states $W_{L}$ and $Z_{L}$ derive their origins and interactions from the symmetry-breaking sector; in particular, heavy Higgs bosons may appear as resonances. The prospects for detecting heavy Higgs bosons, with $m_{H}>2 M_{Z}$, have been analyzed extensively for future $p p$ supercolliders [1]. However, it has been argued that high-energy linear $e^{+} e^{-}$ colliders provide a cleaner experimental environment for a detailed investigation of Higgs-boson characteristics [2]. In the present paper we address the problem of detecting Higgs bosons at possible future $e^{+} e^{-}$machines with center-of-mass (c.m.) energies in the $0.5 \mathrm{TeV}$ range. In a way, our analysis is complementary to Ref. [3], where signals and backgrounds for a very heavy Higgs boson at a $1.5 \mathrm{TeV} e^{+} e^{-}$collider have been studied-neglecting, however, radiative corrections due to bremsstrahlung and beamstrahlung.

In the standard model (SM) there is a single scalar Higgs boson $H$, and the principal $e^{+} e^{-}$production channels are

$$
e^{+} e^{-} \rightarrow Z^{*} \rightarrow Z H \quad(Z H \text { production }),
$$

$$
e^{+} e^{-} \rightarrow \nu \bar{\nu}\left(W^{+}\right)^{*}\left(W^{-}\right)^{*} \rightarrow \nu \bar{\nu} H \quad(W W \text { fusion }),
$$

for which the Feynman diagrams are shown in Fig. 1. The cross section of Eq. (1a) peaks near c.m. energy $\sqrt{s}=M_{Z}+\sqrt{2} m_{H}$ and falls off with increasing energy due to the $s$-channel dependence of the $Z$-boson propagator [4]. Contrariwise, the cross section of Eq. (1b), based on $t$-channel vector-boson exchanges, logarithmically increases with energy and eventually surpasses that of Eq. (1a) [5]. The $Z Z$-fusion mechanism analogous to Eq. (1b) has weaker couplings and hence a much smaller cross section. Figure 2 shows the various cross sections versus $\sqrt{s}$ for $m_{H}=100$ and $150 \mathrm{GeV}$. Results for other $m_{H}$ values can be found in Ref. [6]. It is interesting to note that below the nominal $Z H$-production threshold the $Z H$ mechanism (with a subsequently decaying virtual $Z$ boson) is nevertheless dominant.

At the CERN $e^{+} e^{-}$collider LEP 2, operating at $\sqrt{s}=200 \mathrm{GeV}$, the Higgs-boson discovery limit will be $m_{H} \lesssim 90 \mathrm{GeV}$ assuming high luminosity [7]. An important task of the Next Linear Collider (NLC) is, therefore, the search for an intermediate-mass Higgs boson (IMHB) in the window $M_{Z}<m_{H}<2 M_{W}$. With a total decay width of $\Gamma_{H}<100 \mathrm{MeV}$, an IMHB would be relatively long lived [1]. Thus, the cross sections for the production of an IMHB via Eqs. (1a) or (1b) and its subsequent decay into a certain channel may be simply obtained by multiplying the curves in Fig. 2 by the respective branch- 


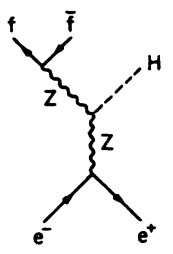

(a)

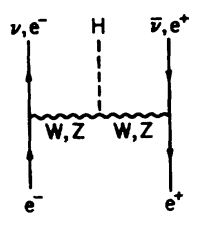

(b)
FIG. 1. Feynman diagrams for Higgs-boson production in $e^{+} e^{-}$collisions via (a) $Z H$ associated production (with subsequent $Z$-boson decay) and (b) $W W$ and $Z Z$ fusions.

ing fraction. In Ref. [8] the branching fractions of an IMHB were studied in detail. We now update that analysis in the following respects. We include electroweak radiative corrections at one loop for all fermionic rates [9]. In addition, we include second-order QCD corrections for the quarkonic widths [10] adopting the physical input parameter $m_{b}=4.25 \mathrm{GeV}$ from Ref. [11]. We also take into account the QCD corrections to the two-gluon mode, which are realized by $H \rightarrow g g(g)$ and $H \rightarrow g q \bar{q}$ and lead to an enhancement by some $60 \%$ [12]. Our results are illustrated in Fig. 3. Figures 2 and 3 summarize the opportunities for an IMHB search at the NLC (up to initial-state radiation corrections that we evaluate below).

In the remainder of this work we shall focus attention on the production of a heavy Higgs boson, with $m_{H}>$ $2 M_{W}$. The dominant SM decay modes are then into four quarks via $H \rightarrow V V \rightarrow q \bar{q} q \bar{q}$ (where $V$ denotes a generic weak boson, $V=W, Z$ ). In our subsequent discussion it will be assumed that the $V V$ pairs are measured in the corresponding four-jet final states $V V \rightarrow j_{1} j_{1} j_{3} j_{4}$, with invariant masses $m\left(j_{1} j_{2}\right)=m\left(j_{3} j_{4}\right)=M_{V}$.

At first sight, the simplest and cleanest heavy Higgs signal appears to be in the dimuon plus four-jet channel

$$
e^{+} e^{-} \rightarrow Z H \rightarrow \mu^{+} \mu^{-} V V
$$

selecting invariant mass $m\left(\mu^{+} \mu^{-}\right)=M_{Z}$; the Higgs-
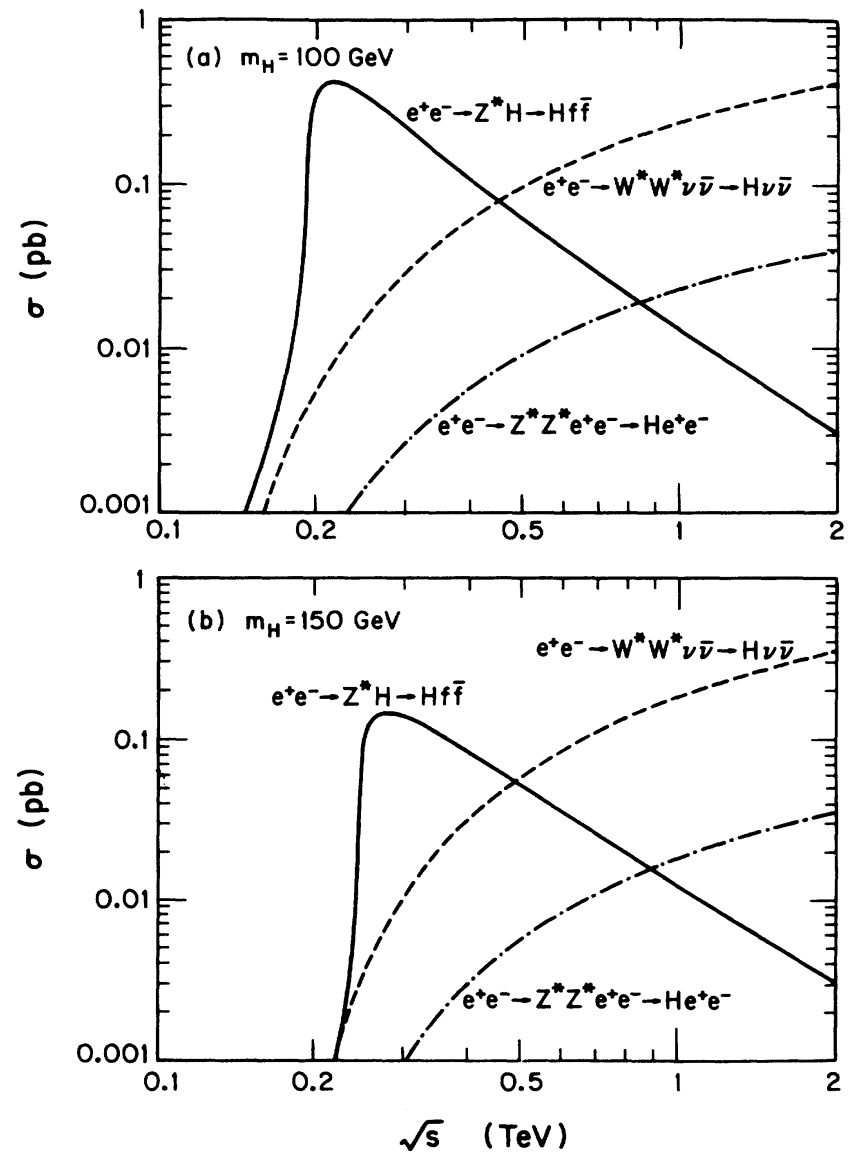

FIG. 2. Cross sections for Higgs-boson production in $e^{+} e^{-}$collisions vs c.m. energy $\sqrt{s}$, assuming (a) $m_{H}=$ $100 \mathrm{GeV}$ and (b) $m_{H}=150 \mathrm{GeV}$. The solid curves represent $Z H$ associated production, allowing for $Z$-boson virtuality below the nominal $Z H$ threshold. The dashed (dot-dashed) curves represent $W W(Z Z)$ fusion.

boson resonance appears here as a peak in the $m_{V V}$ distribution, but the event rate is suppressed by the small branching fraction $B\left(Z \rightarrow \mu^{+} \mu^{-}\right)=0.034$ [13]. It is therefore attractive to investigate also the invisible $Z$ de-
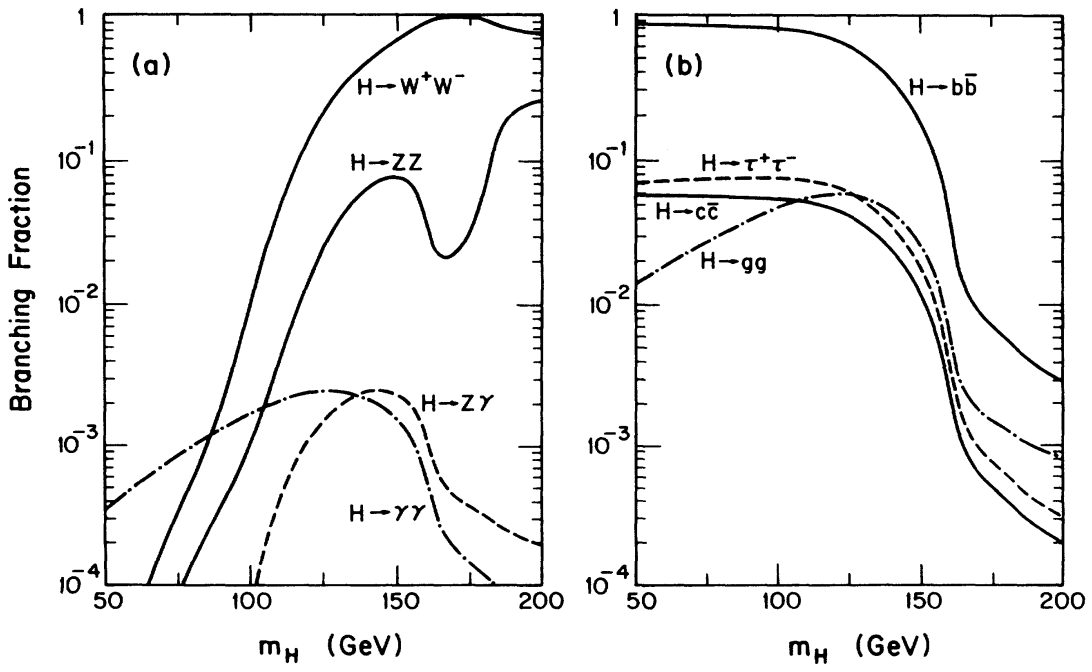

FIG. 3. Branching fractions vs $m_{H}$ for the decays of a Higgs boson into (a) $W W$, $Z Z, Z \gamma$, and $\gamma \gamma$; (b) $b \bar{b}, c \bar{c}, \tau^{+} \tau^{-}$, and $g g$. For the loop-induced decays $m_{t}=130 \mathrm{GeV}$ has been assumed. 
cay to neutrinos, which has a six times larger branching fraction, $\mathrm{B}(Z \rightarrow \nu \bar{\nu})=0.20$ [13]; this leads one to consider the channel

$$
e^{+} e^{-} \rightarrow \nu \bar{\nu} V V
$$

summed over all neutrino flavors, where there is again a resonance peak in $m_{V V}$. The Higgs-boson signal in this channel receives contributions not only from $Z H$ production but also from the $W W$-fusion mechanism. This is both an advantage and a challenge; it further enhances the event rate and also offers the opportunity to separate and compare these contributions and hence to test the relative strengths of the $H Z Z$ and $H W^{+} W^{-}$couplings, predicted by custodial $\mathrm{SU}(2)$ symmetry. The $W W$-fusion contribution to $e^{+} e^{-} \rightarrow \nu \bar{\nu} W^{+} W^{-}(Z Z)$ is not gauge invariant by itself however; the complete set of lowest-order Feynman diagrams for this process is contained in the generic set shown in Fig. 4. The Higgs-boson signal in channel (3) thus has a background from the nonresonant contributions of Fig. 4; it also gets backgrounds from the processes

$$
\begin{aligned}
& e^{+} e^{-} \rightarrow \ell^{+} \ell^{-} W^{+} W^{-}(Z Z), \\
& e^{+} e^{-} \rightarrow \ell^{ \pm} \nu W^{\mp} Z,
\end{aligned}
$$

when one or more charged leptons escape undetected. Processes (3) and (4a) were previously discussed in Ref. [14].

Another channel to consider is

$$
e^{+} e^{-} \rightarrow Z H \rightarrow Z V V \rightarrow 6 \text { jets }
$$

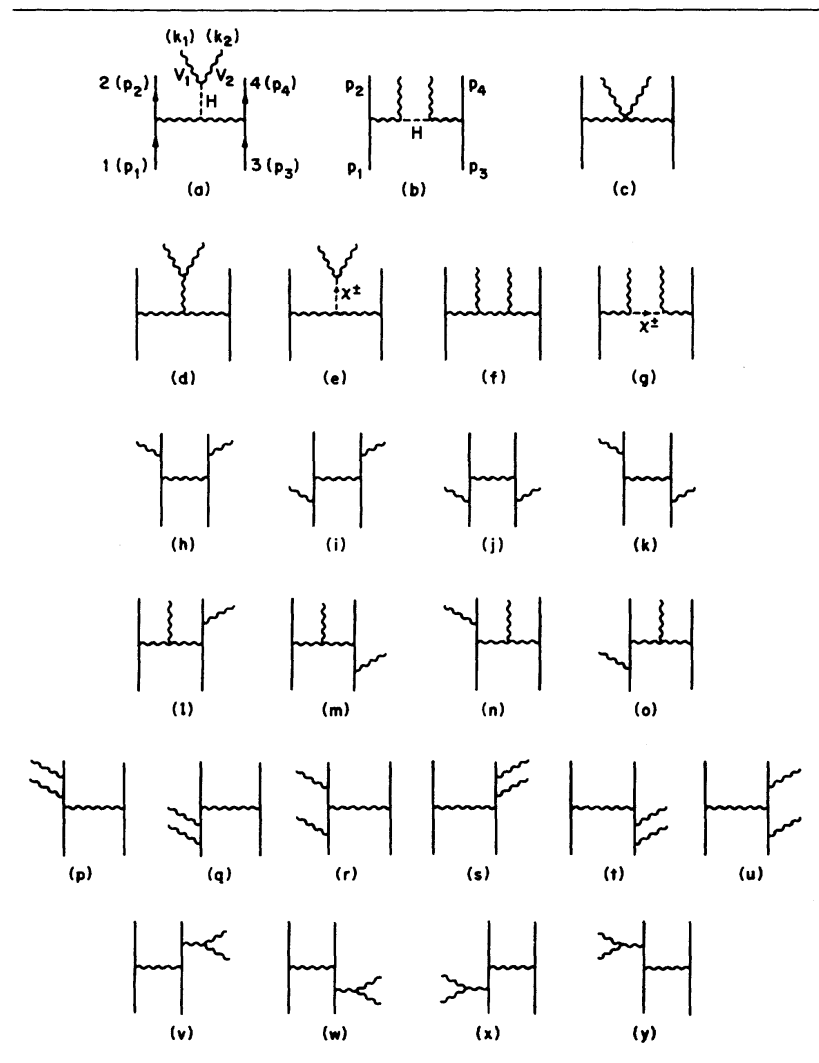

FIG. 4. Generic lowest-order Feynman diagrams for the processes $e^{+} e^{-} \rightarrow \ell_{1} \ell_{2} V_{1} V_{2}$. that benefits from a big branching fraction $B(Z \rightarrow j j)=$ 0.70 [13]. The Higgs-boson signal here appears as a peak in the inclusive $m_{V V}$ spectrum, selecting final states where the jets reconstruct three weak bosons, and summing over all possible $V V$ pairings. This signal is therefore about 20 times bigger than the $\mu \mu j j j j$ signal, or 3.5 times bigger than the $Z H$ component of the $\nu \bar{\nu} j j j j$ signal, and hence is roughly comparable to the total $\nu \bar{\nu} j j j j$ signal. On the other hand, this channel suffers from a large combinatorial background; even if the assignment of $j j$ pairs to weak bosons in a given event happens to be unique, we will have to sum over three possible boson pairings, obtaining signal/background $=1 / 2$ at best. Complications due to jet branchings, jet overlaps, and demarcation between jets are also more critical here than in the four-jet final states, where only the total hadronic invariant mass is ultimately interesting. A proper study of this channel would require detailed jet simulations, that we do not attempt here; however, we can infer the potential signal approximately from our $\mu \mu j j j j$ results.

In addition to intrinsic nonresonant backgrounds and the processes of Eq. (4), significant backgrounds are to be expected from top-quark pair production,

$$
e^{+} e^{-} \rightarrow t \bar{t} \rightarrow\left(b W^{+}\right)\left(\bar{b} W^{-}\right),
$$

that can fake $\nu \bar{\nu} V V, \mu^{+} \mu^{-} V V$, and $Z V V$ final states through various decay modes of the $b$ quarks and $W$ bosons. These backgrounds cannot be precisely predicted until the top quark is discovered, but for the energies of present interest they depend rather weakly on the mass $m_{t}$ in the range $m_{t}=135 \pm 35 \mathrm{GeV}$ indicated by SM studies [15] and consistent with the experimental bound [16] $m_{t}>91 \mathrm{GeV}$. For illustration we shall show the case $m_{t}=150 \mathrm{GeV}$. We ignore backgrounds arising from QCD interactions, where continuum light-quark or gluon jets fake $V \rightarrow j j$ decays; their cross section is suppressed by additional coupling factors $\alpha_{s}^{2}$ and also by the experimental requirement that $m(j j)=m_{V}$.

At high-luminosity $e^{+} e^{-}$colliders, the actual c.m. energy and c.m. frame in which the hard $e^{+} e^{-}$collisions of present interest take place are strongly affected by electromagnetic radiation in the initial state, including both bremsstrahlung (which is universal) and so-called beamstrahlung effects [17] (which depend on properties of the beams near their intersection). Any practical calculation of signals and backgrounds referring to laboratory conditions should therefore take account of these corrections [18].

In the present paper we evaluate the SM Higgs signals and backgrounds in the channels (2)-(6) above, including the contributions of all Feynman diagrams of Fig. 4. We discuss how cleanly the $Z H$-production and $W W$-fusion contributions can be separated in the $\nu \bar{\nu} V V$ channel and how their event rates compare (after selection cuts) with the $Z H$-production signals in the $\mu^{+} \mu^{-} V V$ and six-jet channels. We take the example of an $e^{+} e^{-}$collider with c.m. energy $\sqrt{s}=0.5 \mathrm{TeV}$, which is at the lower end of the energy range $0.5-2.0 \mathrm{TeV}$ currently being considered and is arguably the easiest to achieve. We include effects of initial-state electromagnetic bremsstrahlung plus beamstrahlung, taking typical examples of beam param- 
eters for illustration. After incorporating suitable selection cuts and the initial-state radiation corrections, we conclude that it should be possible to detect a heavyHiggs-boson signal in the $\nu \bar{\nu} W^{+} W^{-}(Z Z)$ channels up to mass $m_{H}=350 \mathrm{GeV}$.

\section{WEAK-BOSON PAIR PRODUCTION IN THE STANDARD MODEL}

We first consider in turn the leading contributions at tree level to the production of weak-boson pairs plus two leptons. An array of generic Feynman diagrams is shown in Fig. 4; a detailed explanation of the diagrams for the various processes is given in Appendix A. The external fermion lines can be labeled $e, \nu$, or $\mu$ and the external weak bosons are labeled $W$ or $Z$. When the external particles are specified, standard selection rules determine the labeling of almost all the other lines. However, a virtual neutral-boson line sometimes represents both $Z$ and $\gamma$, while the flavor of the final neutrinos is sometimes $\nu_{e}$ and sometimes to be summed over $\nu_{e}, \nu_{\mu}$, and $\nu_{\tau}$. The corresponding helicity amplitudes are given in Appendix A, using the techniques of Ref. [19]. All the electron masses are kept finite in the formulas and in the calculations in order to regulate the mass singularities.

The differential cross sections $d \sigma / d m_{V V}$ for the various reactions are shown for the case $m_{H}=200 \mathrm{GeV}$ in Fig. 5; the total integrated cross sections are shown versus $m_{H}$ in Fig. 6. No cuts are imposed here except in the $\mu \mu V V$ channel, where we require

$$
\left|m\left(\mu^{+} \mu^{-}\right)-M_{Z}\right|<15 \mathrm{GeV}
$$

since we are interested only in muon pairs from $Z$ decay. Here and in subsequent figures we omit all smearing effects arising from experimental resolution in measurements of invariant masses and transverse momenta.

The cross section for $e^{+} e^{-} W^{+} W^{-}$production is ex- ceptionally large due to photon exchange processes. The $\nu \bar{\nu} W^{+} W^{-}$channel is particularly sensitive to Higgsboson contributions. The pronounced edges at $m_{H}=$ $2 M_{W}$ and $m_{H}=2 M_{Z}$ in Fig. 6 indicate the onset of the resonance region for the $s$-channel Higgs-boson exchange.

$$
\text { A. } e^{+} e^{-} \rightarrow \nu \bar{\nu} W^{+} W^{-}
$$

The contributing Feynman diagrams are depicted in (a)-(d), (f), (h)-(o), (p), (q), (s), (t), and (v) $-(\mathrm{y})$ of Fig. 4 for the "scattering" channel, where fermion lines 1 and 4 are taken to represent incoming particles, and in (a), (c), (f), (g), (h), (j), and (l)-(y) of Fig. 4 for the "annihilation" channel, where fermion lines 1 and 2 represent incoming particles. The corresponding helicity amplitudes are given in Appendices A 1 and A 2, respectively. Diagrams (a)-(g) contribute via $W^{+} W^{-} \rightarrow$ $W^{+} W^{-}$scattering, but of these only (a) and (b) contain Higgs-boson exchanges and represent the dynamics of the symmetry-breaking sector that we wish to separate. The other diagrams depend on scattering or bremsstrahlung via the standard gauge couplings.

We stress that both the "scattering" and "annihilation" channels of $e^{+} e^{-} \rightarrow \nu \bar{\nu} W^{+} W^{-}$contribute to the Higgs-boson signal. In Fig. 5 the curve for this process includes both contributions; their interference is found to be negligible. Previous calculations at $\sqrt{s}=1.5 \mathrm{TeV}$ [3] neglected all annihilation-channel contributions; this approximation is not justified at $\sqrt{s}=0.5 \mathrm{TeV}$ as Fig. 7(a) illustrates. We note in passing that our calculations of the scattering contribution at $\sqrt{s}=1.5 \mathrm{TeV}$ reproduce the results of Ref. [3].

Figure 7(a) shows separately the "scattering" and "annihilation" contributions to the $d \sigma / d p_{T}$ distribution versus missing transverse momentum $\not_{T}$ for $m_{H}=200 \mathrm{GeV}$. From simple kinematics the distributions terminate at $\left(p_{T}\right)_{\max }=\left(s-4 M_{W}^{2}\right) /(2 \sqrt{s})=224 \mathrm{GeV}$. The lo-
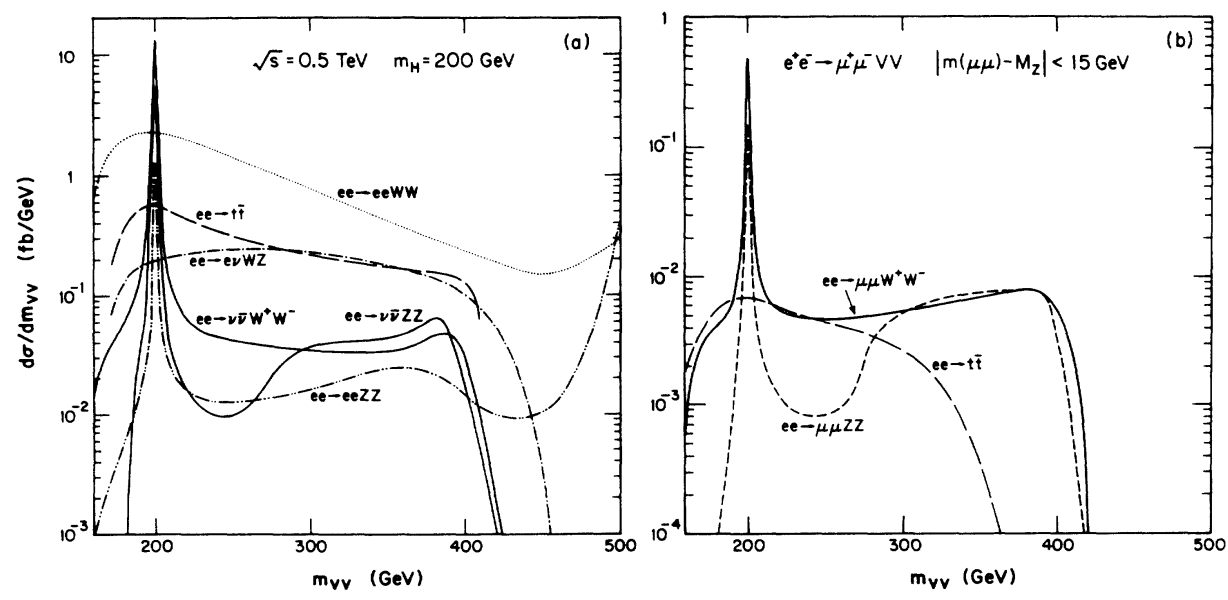

FIG. 5. Differential cross sections $d \sigma / d m_{V V}$ for the production of two leptons plus two weak bosons vs the $V V$ invariant mass $m_{V V}$, where $V=W$ or $Z$, for the case $m_{H}=200 \mathrm{GeV}$. No cuts are imposed apart from the dimuon mass requirement of Eq. (7) in $\mu \mu V V$ channels and the fake- $V$ mass requirement of Eq. (8) in $t \bar{t}$ background cases. All contributions from both scattering and annihilation processes are included: (a) $\nu \bar{\nu} V V$ signal and background channels, (b) $\mu^{+} \mu^{-} V V$ channels. 

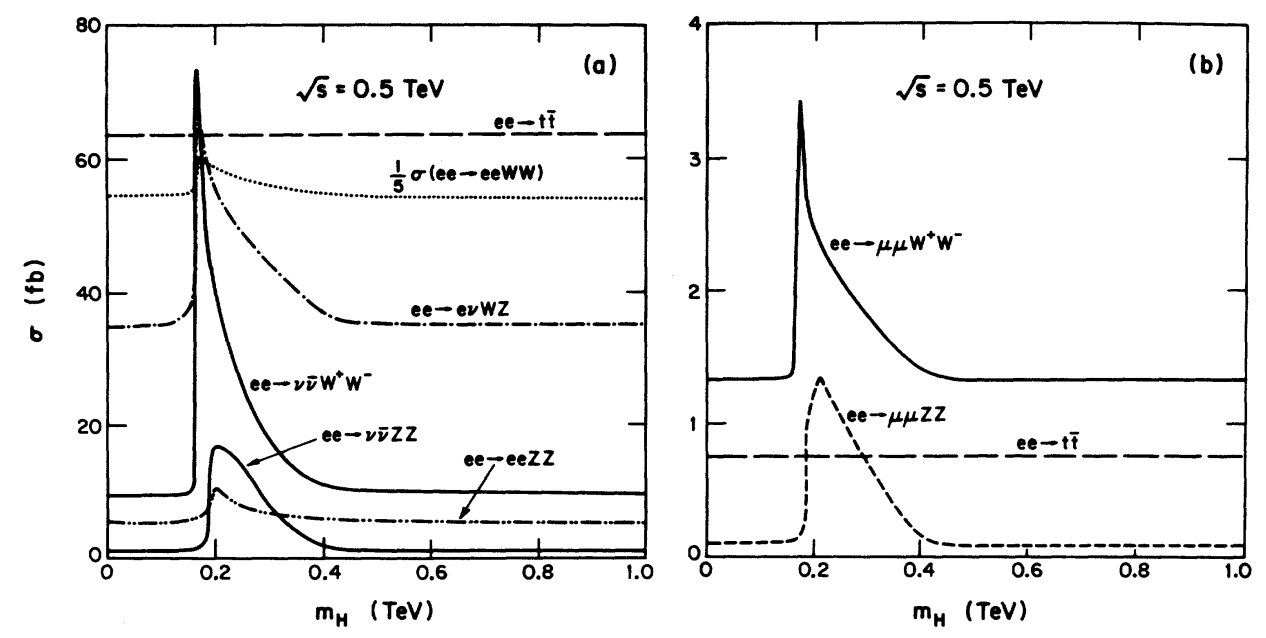

FIG. 6. Total cross sections vs $m_{H}$ : (a) $\nu \bar{\nu} V V$ signal and background channels without cuts, (b) $\mu^{+} \mu^{-} V V$ channels with $\left|m(\mu \mu)-M_{Z}\right|<15 \mathrm{GeV}$. The cross section for $e^{+} e^{-} \rightarrow e^{+} e^{-} W^{+} W^{-}$is relatively large so one-fifth of it is shown. The fake- $V$ mass requirement of Eq. (8) is imposed in the $t \bar{t}$ background cases. The cross sections corresponding to four jets in the final state may be obtained by multiplication with $[B(V \rightarrow j j)]^{2}=0.5$.

cation of the Jacobian peak in the annihilation channel corresponds approximately to the maximum $\not_{T}$ for which the intermediate $Z$ and $H$ bosons in $e^{+} e^{-} \rightarrow$ $Z H \rightarrow \nu \bar{\nu} W W$ are both on mass shell, $\left(\not_{T}\right)_{\text {peak }}=$ $\left\{\left[s-\left(M_{Z}+m_{H}\right)^{2}\right]\left[s-\left(M_{Z}-m_{H}\right)^{2}\right] / s\right\}^{1 / 2} / 2=$ $198 \mathrm{GeV}$. For higher $p_{T}$ one or both bosons are off mass shell, which explains the sharp fall beyond the peak. We conclude that the scattering and annihilation contributions can be separated by their $\not p_{T}$ spectra [or equivalently by their $p_{T}(V V)$ spectra, since $\left|p_{T}\right|=\left|p_{T}(V V)\right|$ in the $\nu \bar{\nu} V V$ final state]. In Fig. 7(b) we also separate the $\not p_{T}$ spectrum of $e^{+} e^{-} \rightarrow \nu \bar{\nu} H$ into the contributions from $W W$-fusion and $Z H$ associated production; see Fig. 1. Now the distributions terminate at $\left(p_{T}\right)_{\max }=\left(s-M_{H}^{2}\right) /(2 \sqrt{s})=210 \mathrm{GeV}$, but the position of the Jacobian peak in the $Z H$ curve is unchanged.
For $m_{H}=200 \mathrm{GeV}$, a $\not p_{T}>175 \mathrm{GeV}$ cut can single out the contribution of the $Z H$ diagram, assuming the c.m. energy to be $0.5 \mathrm{TeV}$. We shall see later that initial-state radiation corrections smear the c.m. energy and with it the $\not_{T}$ dependence; nevertheless, it remains true that $Z H$ production and $V V$ fusion contribute in distinctively different ways and can in principle be separated by a detailed study of the $\not p_{T}$ dependence of the signal.

$$
\text { B. } e^{+} e^{-} \rightarrow \nu \bar{\nu} Z Z
$$

The contributing Feynman diagrams are depicted in (a), (c), and (f)-(u) of Fig. 4 for the scattering channel and in (a), (b), (h) $-(\mathrm{k})$, and (p)-(u) for the annihilation channel with appropriate particle labeling. The helicity amplitudes are given in Appendices A 3 and A 4. This
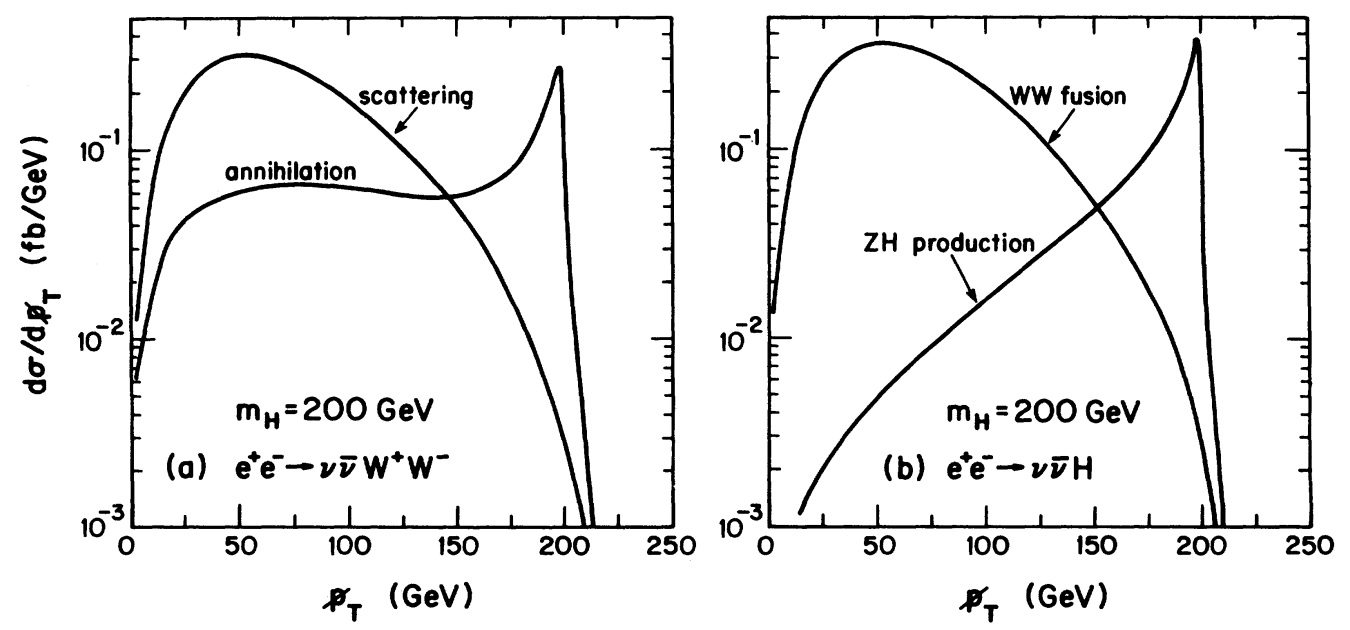

FIG. 7. Differential cross sections without cuts for (a) $e^{+} e^{-} \rightarrow \nu \bar{\nu} W^{+} W^{-}$and (b) $e^{+} e^{-} \rightarrow \nu \bar{\nu} H$ vs the missing transverse momentum $\not_{T}$, assuming $m_{H}=200 \mathrm{GeV}$. Scattering and annihilation contributions are shown separately in (a); $W W$-fusion and $Z H$ production are shown separately in (b). The contributions due to annihilation channels are summed over three neutrino flavors. 
case too has contributions from "scattering" and "annihilation" channels, and both contain the Higgs-boson signal. However, the cross section is much smaller than that for $e^{+} e^{-} \rightarrow \nu \bar{\nu} W^{+} W^{-}$. The major background in this channel arises in the annihilation channel from $e^{+} e^{-} \rightarrow Z H$ with $H \rightarrow Z Z \rightarrow Z \nu \bar{\nu}$ decay [Fig. 4(b)]; it comes from the Higgs boson itself, via a different decay mode from the one we are studying, and gives the broad enhancement at large $m_{V V}$ in Fig. 5(a).

$$
\text { C. } e^{+} e^{-} \rightarrow e^{+} e^{-} W^{+} W^{-}
$$

The contributing Feynman diagrams are depicted in (a), (c), (f), (g), (i), (k), and (l)-(y) of Fig. 4 and explained in Appendix A5. Although this channel has contributions from Higgs-boson-exchange diagrams, it is essentially a background to the Higgs signal, because it is overwhelmingly due to $\gamma$-exchange contributions. In Figs. 5(a) and 6(a) we can see that this background is potentially much bigger than the signal in the $\nu \bar{\nu} W^{+} W^{-}$ channel. But with a suitable $p_{T}(V V)$ cut and centralelectron vetoing, we shall show that this background can be reduced to an unnoticeable level. The calculation of this process together with the process $e^{+} e^{-} \rightarrow$ $\nu \bar{\nu} W^{+} W^{-}$above is almost identical to the case $q \bar{q} \rightarrow$ $W^{+} W^{-} q^{\prime} \bar{q}^{\prime}$ discussed in Ref. [20], where matrix elements are, however, not listed.

$$
\text { D. } e^{+} e^{-} \rightarrow e^{+} e^{-Z Z}
$$

The contributing Feynman diagrams are (a), (b), (h)$(\mathrm{k})$, and (p)-(u) of Fig. 4 with the appropriate substitutions (see Appendix A6). This channel has characteristics similar to Sec. II C, but the cross section is exceedingly small because of the small $Z$ ee coupling.

$$
\text { E. } e^{+} e^{-} \rightarrow e^{ \pm} \nu W^{\mp} Z
$$

The contributing Feynman diagrams are (b)-(r), (t), $(\mathrm{u})$, and (w) $-(\mathrm{y})$ of Fig. 4; the corresponding amplitudes are listed in Appendix A 7. This process gives another background to the signal; the cross section depends on $m_{H}$ because of the Higgs-boson-exchange diagram 4(b). With a $p_{T}(V V)$ cut and central-electron vetoing it can be greatly reduced, as explained below. Furthermore, if it became possible to distinguish accurately between $W$ and $Z$ from the invariant masses of their decay dijets, then this background could be removed.

$$
\text { F. } e^{+} e^{-} \rightarrow \mu^{+} \mu^{-} W^{+} W^{-}(Z Z)
$$

The amplitudes in these cases precisely equal the lepton annihilation amplitudes for the $e^{+} e^{-} \rightarrow$ $e^{+} e^{-} W^{+} W^{-}(Z Z)$ cases, described in Secs. II C and II $\mathrm{D}$ above. In addition to the $H \rightarrow W^{+} W^{-}(Z Z)$ diagrams that give the Higgs-boson mass peaks in the $m_{V V}$ distributions, there are weak-boson scattering and bremsstrahlung diagrams that contribute backgrounds.

In the $\mu^{+} \mu^{-} Z Z$ channel there is also a contribution from $e^{+} e^{-} \rightarrow Z H$ production with $H \rightarrow Z Z \rightarrow \mu^{+} \mu^{-} Z$ decays [Fig. 4(b)]; this appears as a background to the Higgs signal in the $m_{Z Z}$ distribution, if both these $Z$ bosons are assumed to decay hadronically. Since we have here a $Z Z Z$ final state, the event rate in the Higgs peak (and background) could be tripled if we included all three $Z Z$ pairings in the $m_{Z Z}$ distribution. However, this procedure would make sense only if the hadronically decaying $Z$ bosons could be clearly distinguished from $W$ bosons (it would be nonsensical in $\mu^{+} \mu^{-} W^{+} W^{-}$final states) and we do not pursue it here.

$$
\text { G. } e^{+} e^{-} \rightarrow t \bar{t} \rightarrow b \bar{b} W^{+} W^{-} \rightarrow b \bar{b} f_{1} \bar{f}_{2} f_{3} \bar{f}_{4}
$$

We calculate this background process at the tree level, including full spin correlations down to the final $W \rightarrow$ $f_{i} \bar{f}_{j}$ decays into fermions, following the techniques of Ref. [19]. Very heavy quarks decay before they have time to hadronize or depolarize [21], so we treat the $t \rightarrow b W$ decays at the quark level with $100 \%$ branching fraction. When semileptonic $b$ decays are needed, we first hadronize the $b$ quark to a $B$ hadron in the laboratory frame via the Peterson [22] model with parameter $\epsilon=0.05$, consistent with LEP studies [23], and then use the free-quark $b \rightarrow c l \nu$ decay matrix elements following the spectator model of heavy-quark decays. The basic $e^{+} e^{-} \rightarrow t \bar{t}$ cross section is relatively large, of order $700-$ $500 \mathrm{fb}$ for $m_{t}=100-200 \mathrm{GeV}$ at $\sqrt{s}=500 \mathrm{GeV}$.

This final state can be mistaken for $\nu \bar{\nu} V V$ if (a) one $W$ boson decays leptonically $W \rightarrow \ell \nu,(\mathrm{b})$ the charged lepton escapes detection while the neutrino gives large $\phi_{T}$, and (c) the final $b \bar{b}$ pair fakes a $V \rightarrow j j$ decay. This background can be reduced by requiring large $\not_{T}$ and by vetoing central leptons, the same criteria that suppress the other backgrounds. We note that leptonic $\tau$ decays give final $e$ or $\mu$ while hadronic $\tau$ decays give identifiable narrow jets; although the technical details for vetoing central $\tau$ 's must differ from those for vetoing central electrons and muons, we shall here treat them all the same as a first approximation. In Figs. 5(a) and 6(a) we show the potential $t \bar{t}$ background in the $\nu \bar{\nu} V V$ channel, before making selective cuts, for the case $m_{t}=150 \mathrm{GeV}$. We first calculate the cross section for $\ell \nu j j j j$ production, summing the contributions from $\ell=e, \mu, \tau$, and requiring that the fake $V$ generated by $b$ and $\bar{b}$ jets has invariant mass within $15 \mathrm{GeV}$ of the $W$ or $Z$ mass:

$$
M_{W}-15 \mathrm{GeV}<m(b \bar{b})<M_{Z}+15 \mathrm{GeV} \text {. }
$$

This cross section is divided by the square of the branching fraction $B(V \rightarrow j j)=0.70$ to convert it to an effective $\nu \bar{\nu} V V$ cross section. Then $m_{V V}$ denotes the invariant mass of the hadronically decaying $W$ plus the fake $V$.

These $t \bar{t}$ final states faking $\nu \bar{\nu} V V \rightarrow \nu \bar{\nu} j j j j$ all have the property that three of the jets have invariant mass $m(j j j)=m_{t}$. We could therefore in principle suppress this background completely by vetoing final states where any three jets satisfy $\left|m(j j j)-m_{t}\right|<15 \mathrm{GeV}$, say, once the top quark has been discovered and $m_{t}$ is known. The price paid would be the loss of some of the $H \rightarrow V V \rightarrow j j j j$ signal, but this price varies greatly according to the values of $m_{t}$ and $m_{H}$. If $m_{t}=150 \mathrm{GeV}$, 
for example, this invariant mass veto would destroy about $90 \%(70 \%)$ of the Higgs-boson signal for $m_{H}=200 \mathrm{GeV}$ $(250 \mathrm{GeV})$, which is probably too much to pay. But if $m_{t}=100 \mathrm{GeV}$, the corresponding Higgs signals would be reduced by only $45 \%(20 \%)$ instead, while for $m_{H}=300$ or $350 \mathrm{GeV}$ the loss of signal would be less than $10 \%$. All this assumes of course that the eventual detectors would allow sufficiently accurate multijet invariant mass reconstructions. In our present analysis we do not apply this three-jet mass veto, since it would introduce a confusing multiplicity of cases to discuss; however, it remains a potentially helpful cut for future application.

Top-quark pair production can also be mistaken for $\mu \mu V V$ production if two of the heavy flavors decay into muons. Two primary top semileptonic decays give the wrong jet multiplicity, but one primary $t$ decay plus one secondary $b$ decay to muons can fake $\mu \mu V V$ if the hadronic debris from the two $b$ quarks (after one semileptonic decay) combines to fake $V \rightarrow j j$. With smaller probability, two secondary $b$ decays to muons can also fake $\mu \mu V V$, if the hadronic debris from the $b$ quarks does not obscure the two remaining genuine $W$ bosons; we shall ignore this contribution and other small effects arising from semileptonic charm decays. These backgrounds can be suppressed by requiring the dimuon invariant mass to be close to $M_{Z}$ and by vetoing events with large $p_{T}$. They can be suppressed further by adding some isolation requirement on the muons, since $Z \rightarrow \mu \mu$ typically gives isolated muons whereas $b$ and $c$ decays give muons in or near jets, but we do not pursue this further here.

In Figs. 5(b) and 6(b) we show the potential $t \bar{t}$ background in the $\mu \mu V V$ channel, before making selective cuts, for the case $m_{t}=150 \mathrm{GeV}$. We calculate the cross section for $\mu \mu j j j j$ production, requiring that the dimuon invariant mass satisfies the constraint Eq. (7) and that the fake $V$ from $b+\bar{b}$ hadronic debris satisfies the constraint Eq. (8). This cross section is divided by the square of the branching fraction $B(V \rightarrow j j)=0.70$, to reduce it to an effective $\mu \mu V V$ cross section. Then $m_{V V}$ denotes the invariant mass of one true $W$ plus one fake $V$, as before.

Finally, $t \bar{t}$ production can also be mistaken for $Z V V \rightarrow$ 6-jet production if all the $W$ bosons and $B$ hadrons decay hadronically and the two $b$ jets fake a $V \rightarrow j j$ configuration. This background is suppressed a little by the decay branching fractions and rather more by the constraint $m(b \bar{b}) \approx M_{V}$. It could be suppressed completely by vetoing $m(j j j) \approx m_{t}$ configurations, as discussed above, but we do not apply this veto in our present work.

\section{RESULTS AFTER KINEMATIC CUTS}

At $\sqrt{s}=0.5 \mathrm{TeV}$ there are seldom any events for $W$ bosons having absolute rapidity $|y|>1.5$, and there is not much difference between rapidity cuts of 1.0 and 1.5. Furthermore, experimental acceptance will force some such cut upon us anyway, since jet measurements will be impossible close to the beam axis. In our calculations we shall therefore make the cut

$$
|y(V)|<1
$$

to approximate the experimental acceptance, with no serious loss in signal.

In the $\nu \bar{\nu} V V$ channel, the backgrounds from $e^{+} e^{-} \rightarrow$ $e^{+} e^{-} W^{+} W^{-}$(mainly due to $\gamma$-exchange processes), from $e^{+} e^{-} \rightarrow e \nu W Z$, and from $e^{+} e^{-} \rightarrow t \bar{t}$ are potentially dangerous; see Figs. 5 and 6 . Our strategy to reduce the first two backgrounds is similar to that in Ref. [3]. We first suppress the contribution from the $\gamma$-exchange poles by a cut

$$
p_{T}(V V)>45 \mathrm{GeV},
$$

which effectively removes the double- $\gamma$ pole contribution to $e^{+} e^{-} \rightarrow e^{+} e^{-} W^{+} W^{-}$. In addition, we veto events with a visible electron. We assume that $e^{ \pm}$will be identifiable if they have high energy and are emitted in the central region (neglecting the possibility of losing electrons in jets); we therefore veto all events that contain $e^{ \pm}$with

$E_{e^{ \pm}}>50 \mathrm{GeV}$ and $\left|\cos \left(\theta_{e^{ \pm}}\right)\right|<\cos (0.15)$.

This proves to be very effective in reducing the $e^{+} e^{-} W^{+} W^{-}$background, but less in suppressing the $e \nu W Z$ background. The $t \bar{t}$ background is little suppressed by the $p_{T}(V V)$ cut but is considerably reduced by vetoing all central leptons; for this we extend the criteria of Eq. (11) to apply to all charged leptons $e, \mu$, and $\tau$. Our cuts are very similar to the ones used in Ref. [3].

The results for $\nu \bar{\nu} V V$ signal and background channels are summarized in Fig. 8 for the differential cross sec-

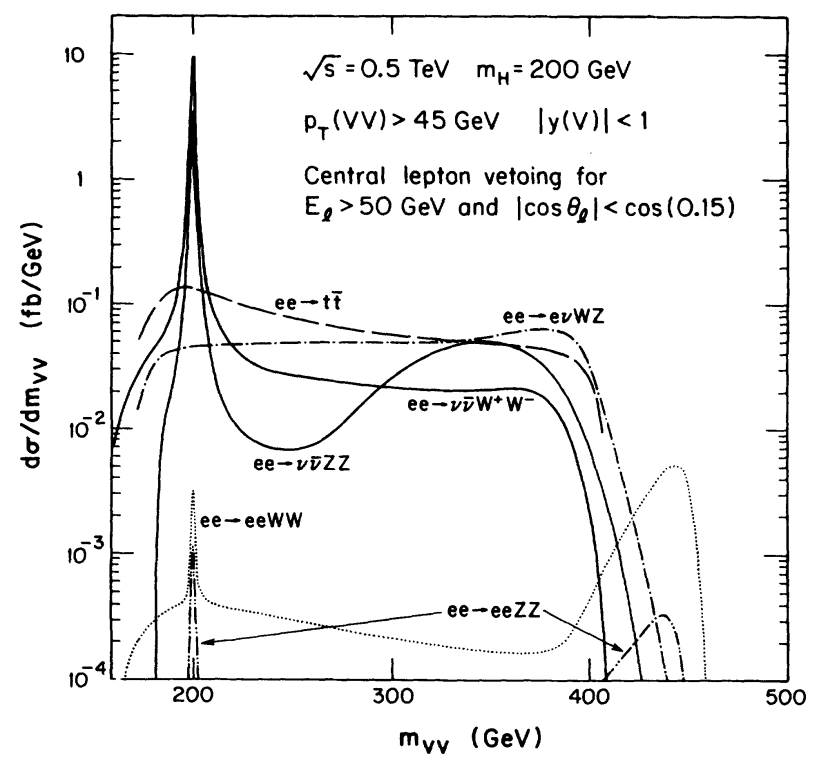

FIG. 8. Contributions to the $\nu \bar{\nu} V V$ signals and backgrounds, with acceptance cuts but without initial-state radiative corrections, for the case $m_{H}=200 \mathrm{GeV}$. Differential cross sections are shown for $e^{+} e^{-} \rightarrow \nu \bar{\nu}\left(e^{+} e^{-}, e \nu\right) V V$ vs the $V V$ invariant mass $m_{V V}$, where $V=W, Z$; here $|y(V)|<1$, $p_{T}(V V)>45 \mathrm{GeV}$, and central $e^{ \pm}$vetoing by $E_{e^{ \pm}}>50 \mathrm{GeV}$ and $\left|\cos \left(\theta_{e^{ \pm}}\right)\right|<\cos (0.15)$ has been applied. These curves receive contributions from both scattering and annihilation channels. The background from $e^{+} e^{-} \rightarrow t \bar{t}$ production is also shown, with the fake- $V$ mass constraint of Eq. (8) plus the corresponding $p_{T}(V V)$ and $|y(V)|$ cuts and a veto on all central leptons $e, \mu, \tau$. 
tion versus the invariant mass $m_{V V}$ of the weak-boson pair, and in Fig. 9 for the total cross section versus $m_{H}$; see also upper entries of Table I. With the above cuts the total cross sections for $e^{+} e^{-} \rightarrow e^{+} e^{-} W^{+} W^{-}$and $e^{+} e^{-} Z Z$ are now exceedingly small, being of order 0.1 and $0.01 \mathrm{fb}$, respectively. We see that there is an excellent Higgs-boson signal with little background, especially for the mass range $160 \mathrm{GeV}<m_{H}<225 \mathrm{GeV}$, where the $H \rightarrow W W, Z Z$ resonance signal exceeds $20 \mathrm{fb}$ with a total nonresonant background of about $40 \mathrm{fb}$. For an assumed annual integrated luminosity of $50 \mathrm{fb}^{-1}, 2$-jet branching fractions $B(W \rightarrow j j) \approx B(Z \rightarrow j j)=0.7$, and perhaps $50 \%$ instrumental efficiency, a $20 \mathrm{fb}$ signal translates into 250 signal events per year in the $H \rightarrow V V \rightarrow 4$ jet channels. It should be possible to detect much smaller signals than this.

Further details of the $e^{+} e^{-} \rightarrow \nu \bar{\nu} V V$ signals and backgrounds are given in the upper entries of Table I, where total cross sections are listed for a range of Higgsboson mass values (excluding bremsstrahlung and beamstrahlung corrections, as do all our calculations so far). The intrinsic background in the $\nu \bar{\nu} W W$ channel, i.e., the cross section without the resonance peak in $m_{W W}$, may be estimated by calculating with some small Higgsboson mass, $m_{H}=50 \mathrm{GeV}$ say; this approximation agrees closely with the heavy-Higgs-boson calculations outside the resonance peak and interpolates smoothly under the peak. Subtracting this background from the total $\nu \bar{\nu} W W$ cross section gives an estimate of the Higgs signal in this channel. The sum of the intrinsic and other (misidentification) backgrounds gives the total integrated background. In the $\nu \bar{\nu} Z Z$ channel, the principal intrinsic background comes from $Z H$ production with $H \rightarrow Z \nu \bar{\nu}$ decay, which cannot be obtained from any light- $H$ calculation; a rough estimate of this background is provided by taking $2 / 3$ of the integrated $\nu \bar{\nu} Z Z$ production, with the remaining $1 / 3$ being due to the signal. Note however that for discussions of the significance of each signal, we should compare with the part of the background lying

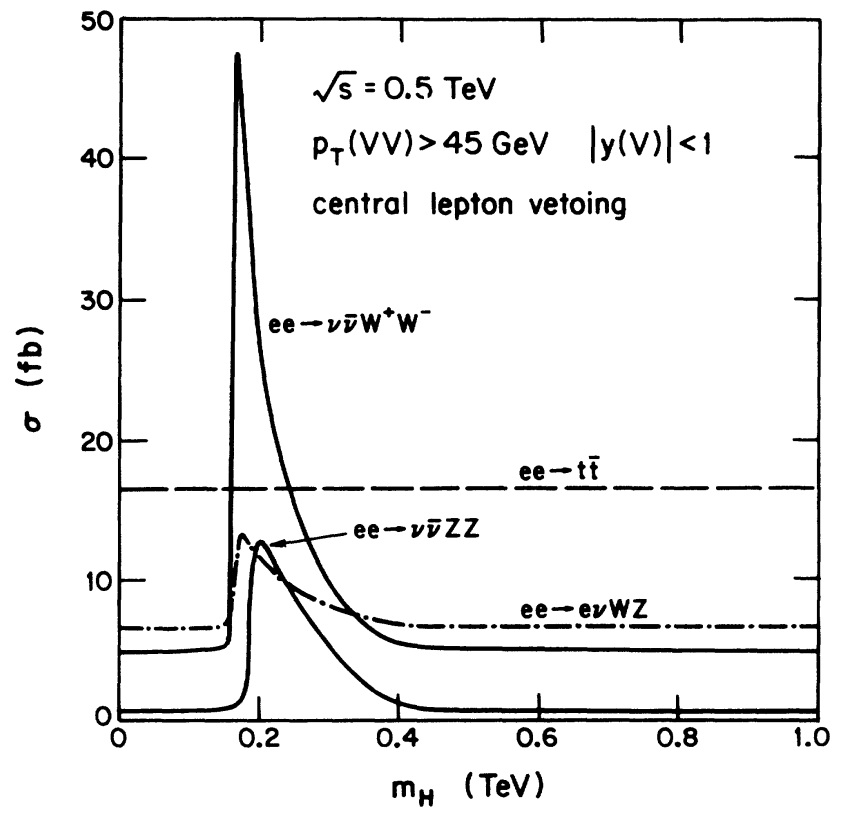

FIG. 9. Total cross sections for the $\nu \bar{\nu} V V$ signals and backgrounds vs $m_{H}$ from $e^{+} e^{-} \rightarrow \nu \bar{\nu} W^{+} W^{-}, \nu \bar{\nu} Z Z$, $e \nu W^{ \pm} Z$, and $t \bar{t}$, imposing the same cuts as in Fig. 8. The $e^{+} e^{-} \rightarrow e^{+} e^{-} W^{+} W^{-}$and $e^{+} e^{-} Z Z$ contributions after cuts are extremely small, of order 0.1 and $0.01 \mathrm{fb}$.

under the resonance peak that is an order of magnitude smaller than the integrated background in each channel given by our tables.

The Higgs-boson signals in the $Z H \rightarrow \mu \mu V V$ channels do not suffer from potentially large backgrounds and therefore do not need strong cuts for their extraction. However, the $t \bar{t}$ background has typically large $p_{T}=p_{T}(V V)$ due to the leptonic $W$-boson decay, unlike the signal or the other backgrounds; we therefore choose to suppress $t \bar{t}$ contributions by requiring

$$
\not p_{T}<40 \mathrm{GeV} \text {. }
$$

TABLE I. Cross sections in fb for the various channels contributing to the $\nu \bar{\nu} V V$ Higgs-boson signals and backgrounds, demanding $|y(V)|<1, p_{T}(V V)>45 \mathrm{GeV}$, and vetoing central leptons [with $E_{\ell^{ \pm}}>50 \mathrm{GeV}$ and $\left|\cos \theta_{\ell^{ \pm}}\right|<\cos (0.15)$ ]. For each channel and value of $m_{H}$, the upper entry does not include the effects of beamstrahlung and bremsstrahlung. In the lower entry, these effects are taken into account for the Palmer $G$ (DESY/Darmstadt narrow-band) design. The corresponding numbers for the continuum production of $\nu \bar{\nu} W W$, simulated by the choice $m_{H}=50$ $\mathrm{GeV}$, are $5,2.9$ (4.5). Subtracting this from the $\nu \bar{\nu} W W$ result leaves the corresponding Higgs-boson signal. In the $\nu \bar{\nu} Z Z$ channel, the signal is about $\frac{1}{3}$ of the given cross section. The numbers for the $t \bar{t}$ background are 16, 31 (19), assuming $m_{t}=150 \mathrm{GeV}$.

\begin{tabular}{lccccc}
\hline \hline & $m_{H}=175 \mathrm{GeV}$ & $m_{H}=200$ & $m_{H}=250$ & $m_{H}=300$ & $m_{H}=350$ \\
\hline$\nu \bar{\nu} W W$ & 42 & 29 & 16 & 9.8 & 6.9 \\
& $31(42)$ & $18(25)$ & $8.9(14)$ & $5.0(8.9)$ & $3.5(5.9)$ \\
$\nu \bar{\nu} Z Z$ & 1.3 & 13 & 8.8 & 5.4 & 2.7 \\
& $0.62(0.83)$ & $6.9(9.5)$ & $4.0(6.1)$ & $1.8(3.5)$ & $0.69(1.6)$ \\
$e^{+} e^{-} W W$ & 0.15 & 0.15 & 0.14 & 0.13 & 0.12 \\
& $0.15(0.15)$ & $0.16(0.16)$ & $0.15(0.16)$ & $0.15(0.16)$ & $0.15(0.15)$ \\
$e^{+} e^{-} Z Z$ & 0.016 & 0.014 & 0.01 & 0.009 & 0.009 \\
& $0.08(0.02)$ & $0.05(0.02)$ & $0.016(0.015)$ & $0.011(0.011)$ & $0.008(0.011)$ \\
$e \nu W Z$ & 13 & 12 & 9.4 & 8.5 & 7.4 \\
& $13(14)$ & $9.2(10)$ & $6.6(8.5)$ & $4.8(7.4)$ & $4.1(6.7)$ \\
\hline \hline
\end{tabular}


In addition to the $\mu^{+} \mu^{-}$mass constraint of Eq. (7) and the fake- $V$ mass constraint of Eq. (8), we also impose the rapidity cut of Eq. (9), approximating the likely experimental acceptance, both on the vector bosons and on the $\mu^{+} \mu^{-}$system. The total cross section and $m_{V V}$ dependence differ little from the uncut distributions shown in Figs. $5(\mathrm{~b})$ and $6(\mathrm{~b})$ (apart from $t \bar{t}$ contributions), so we do not plot them again. Details of the integrated $\mu \mu V V$ signals and backgrounds are given in Table II. In the $\mu \mu W W$ channel the background is estimated by calculating with a light Higgs boson, as in the $\nu \bar{\nu} W W$ channel (Table I). In the $Z Z Z \rightarrow \mu \mu Z Z$ channel, where the final $Z Z$ are assumed to decay into four jets, the principal background comes from $H \rightarrow Z Z \rightarrow \mu \mu j j$ decays; this accounts for about $2 / 3$ of the integrated cross section while the remaining $1 / 3$ comes mostly from the $H \rightarrow Z Z \rightarrow j j j j$ signal. We note that the net signal cross sections in the $\mu \mu V V$ channels (Table II) are about 10-20 times smaller than those in the $\nu \bar{\nu} V V$ channels (Table I).

The net Higgs signal in the $Z H \rightarrow 6$-jet channel is related to that in the $Z H \rightarrow \mu \mu j j j j$ channel by the ratio of branching fractions $B(Z \rightarrow j j) / B\left(Z \rightarrow \mu^{+} \mu^{-}\right) \approx 20$, as remarked in Sec. I, if we continue to apply essentially the same acceptance cuts both to the muons and to the jets. We can therefore use the latter signal to estimate the former; folding in the $V \rightarrow j j$ branching fractions, we see from Table II that the Higgs-boson signal in 6-jet final states falls from about $15 \mathrm{fb}$ at $m_{H}=175 \mathrm{GeV}$ to about $3 \mathrm{fb}$ at $m_{H}=350 \mathrm{GeV}$. The $e^{+} e^{-} \rightarrow V V V \rightarrow 6$-jet background is harder to estimate; it depends on detailed questions of jet resolution, etc. However, the minimal combinatorial background from the $Z H \rightarrow Z V V$ process is at least twice the signal. Also, we find that the effective $t \bar{t} \rightarrow V V V \rightarrow 6$-jet background (with $b \bar{b}$ faking a $V$ ) is of order $75 \mathrm{fb}$ for $m_{t}=150 \mathrm{GeV}$, if we simply impose the mass and rapidity cuts of Eqs. (8) and (9). Compared to the $\nu \bar{\nu} V V$ case, the signals in this channel have similar strength but the backgrounds are much bigger and more problematical.

Finally we recall that the effects of experimental resolution, arising from calorimeter fluctuations, semileptonic decays in jets, etc., remain to be added to our present calculations. These effects will somewhat smear the sharp peaks in invariant masses and transverse mo- menta that we show. There are also important corrections from initial-state radiation, that we now discuss.

\section{EFFECTS OF BREMSSTRAHLUNG AND BEAMSTRAHLUNG}

It is well known that cross sections measured in highenergy $e^{+} e^{-}$collisions are greatly affected by QED radiative corrections. (However, we can as a first approximation neglect weak corrections, which are known to vary between $-7 \%$ and $+6 \%$ for the $e^{+} e^{-} \rightarrow Z H$ cross section at $\sqrt{s}=0.5 \mathrm{TeV}[24]$.) In general, the main effect is due to bremsstrahlung from the initial state, which lowers the effective c.m. energy available in the main process and leads to a typical smearing of the distributions in the subprocess c.m. energy $\sqrt{\hat{s}}$. The size of the effect can be easily estimated by considering the large leading logarithms. In $O\left(\alpha^{n}\right)$ they are of the form $(\alpha / \pi)^{n} \ln ^{n}\left(s / m_{e}^{2}\right)$ $(n=1,2, \ldots)$, which follows from Sudakov's theorem [25]. In an inclusive experiment there are no similar logarithms due to final-state particles. This is guaranteed by the Kinoshita-Lee-Nauenberg theorem [26], which states that mass singularities associated with outgoing particles cancel when all final states with the same invariant mass are summed up. For $\sqrt{s}=0.5 \mathrm{TeV}$ one has $(\alpha / \pi) \ln \left(s / m_{e}^{2}\right) \approx 7 \%$. It is, therefore, clear that any predictions for processes at the NLC that ignore initialstate bremsstrahlung will vary from crude to completely inadequate. By analogy to the situation at the $Z$ peak it is clear that in the presence of a not-too-broad Higgs resonance even a rigorous treatment to $O(\alpha)$ would still fail to lead to reliable predictions. An $O\left(\alpha^{2}\right)$ calculation in connection with soft-photon exponentiation [27] would be in order. Unfortunately, full $O\left(\alpha^{2}\right)$ results are only available for the class of processes where the electron-positron pair annihilates into a neutral gauge boson [28]. However, the pattern in which the leading logarithms arrange themselves is universal for all reactions with an $e^{+} e^{-}$initial state. Leading-order initialstate bremsstrahlung is most conveniently included by convoluting the reduced cross section with exponentiated splitting functions for the incident electron and positron beams [29]. These splitting functions characterize the probability of finding an electron (positron) with a given longitudinal-momentum fraction inside the original elec-

TABLE II. Cross sections in fb for the $\mu^{+} \mu^{-} V V$ channels after imposing the cuts $|y(V)|,\left|y\left(\mu^{+} \mu^{-}\right)\right|<1,\left|m\left(\mu^{+} \mu^{-}\right)-M_{Z}\right|<15 \mathrm{GeV}, p_{T}<40 \mathrm{GeV}$. For each channel and value of $m_{H}$, the upper entry does not include the effects of beamstrahlung and bremsstrahlung. In the lower entry, these effects are taken into account for the Palmer $G$ (DESY/Darmstadt narrow-band) design. The corresponding numbers for the continuum production of $\mu \mu W W$, simulated by the choice $m_{H}=50 \mathrm{GeV}$, are $0.64,0.26(0.55)$. Subtracting this from the $\mu \mu W W$ result leaves the corresponding Higgs-boson signal. In the $\mu \mu Z Z$ channel, the signal is about $\frac{1}{3}$ of the given cross section. The numbers for the $t \bar{t}$ background are $0.26,0.23(0.26)$, assuming $m_{t}=150 \mathrm{GeV}$.

\begin{tabular}{cccccc}
\hline \hline & $m_{H}=175 \mathrm{GeV}$ & $m_{H}=200$ & $m_{H}=250$ & $m_{H}=300$ & $m_{H}=350$ \\
\hline$\mu \mu W W$ & 2.2 & 1.5 & 1.2 & 1.0 & 0.82 \\
& $0.87(1.8)$ & $0.71(1.4)$ & $0.54(1.1)$ & $0.41(0.87)$ & $0.31(0.7)$ \\
$\mu \mu Z Z$ & 0.11 & 1.2 & 0.86 & 0.56 & 0.28 \\
& $0.19(0.13)$ & $0.57(1.0)$ & $0.42(0.8)$ & $0.24(0.51)$ & $0.092(0.24)$ \\
\hline \hline
\end{tabular}


tron (positron) after multiple-photon emission and can be obtained by solving QCD-like evolution equations. In our analysis we adopt Eq. (20) of the first paper of Ref. [29] neglecting the numerically insignificant contribution of those $\beta^{2}$ terms which do not participate in the exponentiation. For $e^{+} e^{-}$reactions in the continuum it is evident that initial-state bremsstrahlung should tend to reduce (enhance) a cross section which is increasing (decreasing) with increasing energy.

A completely novel feature which will be faced at the NLC is the so-called beamstrahlung phenomenon [17], which is an unavoidable consequence of the quest for luminosities which exceed current achievements by 3 orders of magnitude. It occurs when particles in one bunch undergo bremsstrahlung upon entering the electromagnetic field of the other bunch. These particles thus interact coherently with a sizable part of the opposite bunch. The intensity of the emitted beamstrahlung therefore increases with the strength of the fields generated by the bunches, which in turn grows with the particle density of the bunches and hence with the luminosity per bunch crossing. Beamstrahlung effects delicately depend on the details of the machine operation and a realistic estimate of their characteristics can only be obtained through Monte Carlo simulation. In our analysis we generate beamstrahlung (and bremsstrahlung) events using the program package BEAMSPEC by Barklow [30].

The optimization of a linear-collider design proceeds in a multidimensional parameter space with a network of constraints [31]. The currently existing concepts for a $500 \mathrm{GeV}$ NLC fall into three broad categories [32]. (1) SLAC and KEK propose a traveling-wave copper structure at room temperature, operating at $11.4 \mathrm{GHz}(X$ band), and a gradient of $50-100 \mathrm{MV} / \mathrm{m}$. There is an option with high luminosity but strong beamstrahlung (Palmer $G$ ) and an alternative with moderate beamstrahlung at the cost of a factor 2-4 in luminosity (Palmer F) [31]. (2) DESY/Darmstadt proposals extend the present SLAC Linear Collider (SLC) technology to higher energies, using a warm traveling-wave copper structure, operating at $2.8 \mathrm{GHz}$, and a gradient of $17 \mathrm{MV} / \mathrm{m}$. The original wideband version [33] suffers from a large intrinsic linac energy spread, which impairs the resolving power for very narrow structures. This drawback has been largely removed in the narrow-band version [34]. (3) The Cornell TeV Energy Superconducting Linear Accelerator (TESLA) design [35] proposes to use a superconducting standing-wave radio-frequency structure at $1.3 \mathrm{GHz}$ with a gradient of $25 \mathrm{MV} / \mathrm{m}$. Thanks to very long bunches and large spot sizes, beamstrahlung is reduced to a level comparable to initial-state bremsstrahlung.

It has become customary [36] to characterize the beamstrahlung spectrum by a dimensionless beamstrahlung parameter $\Upsilon$, which is defined by $\Upsilon=\gamma B / B_{c}$, where $\gamma=$ $E_{b} / m_{e} c^{2}$ is the ratio of the initial beam energy to the electron mass, $B$ is the average magnetic field inside a bunch, and $B_{c}=m_{e}^{2} c^{3} / e \hbar=4.4 \times 10^{13} \mathrm{G}$ is the Schwinger critical field. For $\Upsilon<1$, the beamstrahlung energy loss is a monotonically increasing function of $\Upsilon$ [31]. For the above designs the numbers are 0.385 (Palmer $G$ ), 0.108 (Palmer F), 0.065 (DDwb), 0.013 (DDnb), and 0.008
(TESLA) [32]. To start with, we consider the Palmer $G$ design to mark the most disadvantageous scenario. Figures 10-13 and the lower entries in the tables display the characteristic features of bremsstrahlung and beamstrahlung for a collider of this type. For comparison, we then repeat the central parts of our analysis assuming the more favorable DESY/Darmstadt narrow-band design; see Figs. 14, 15 and parenthesized entries in the tables. The corresponding results for TESLA are very similar to those for DESY/Darmstadt.

We first illustrate the implications for intermediateand high-mass Higgs-boson production rates. Figure 10 shows the $e^{+} e^{-} \rightarrow \nu \bar{\nu} H$ cross sections via the $Z H$ production and $W W$-fusion subprocesses versus $m_{H}$ at $\sqrt{s}=0.5 \mathrm{TeV}$, before and after corrections for bremsstrahlung and beamstrahlung. We see that these corrections enhance the $Z H$-production mechanism for $m_{H} \lesssim 200 \mathrm{GeV}$; here the increase in cross section from smearing to lower c.m. energy wins over threshold effects, but for higher $m_{H}$ the threshold suppression takes over. Production via $W W$ fusion is reduced for all $m_{H}$ values, since the cross section falls monotonically as $\sqrt{\hat{s}}$ is decreased.

Figure 11 illustrates some characteristic features of beamstrahlung and bremsstrahlung on the $\nu \bar{\nu} H$ signals from $W W$ fusion and $Z H$ associated production, assuming $m_{H}=200 \mathrm{GeV}$. Figure 11 (a) shows the $\sqrt{\hat{s}}$ dependence after these corrections are taken into account; without corrections the entire process would occur at $\sqrt{\hat{s}}=0.5 \mathrm{TeV}$. As expected, the $W W$-fusion process, which has a cross section rising with $\hat{s}$, contributes mostly at the top of the $\hat{s}$ range; the $Z H$-production process,

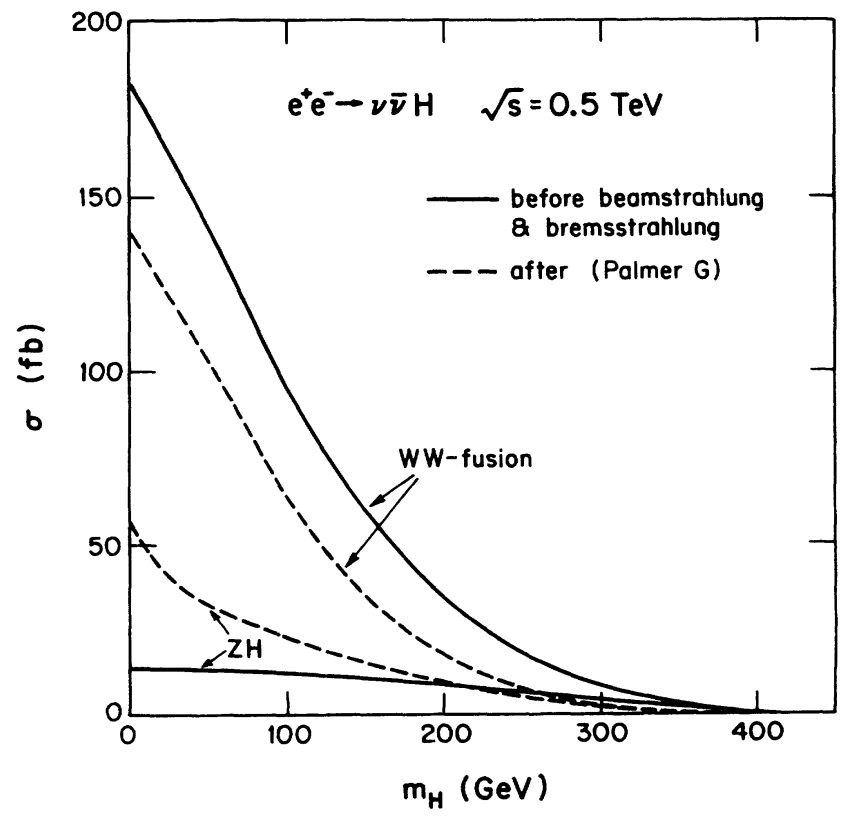

FIG. 10. Total cross sections at $\sqrt{s}=500 \mathrm{GeV}$ for $e^{+} e^{-} \rightarrow \nu \bar{\nu} H$, before and after bremsstrahlung and beamstrahlung (Palmer $G$ ) corrections, as a function of $m_{H}$. Both $W W$-fusion and $Z H$-production channels are shown. Dashed (solid) curves denote results with (without) QED radiative corrections. 

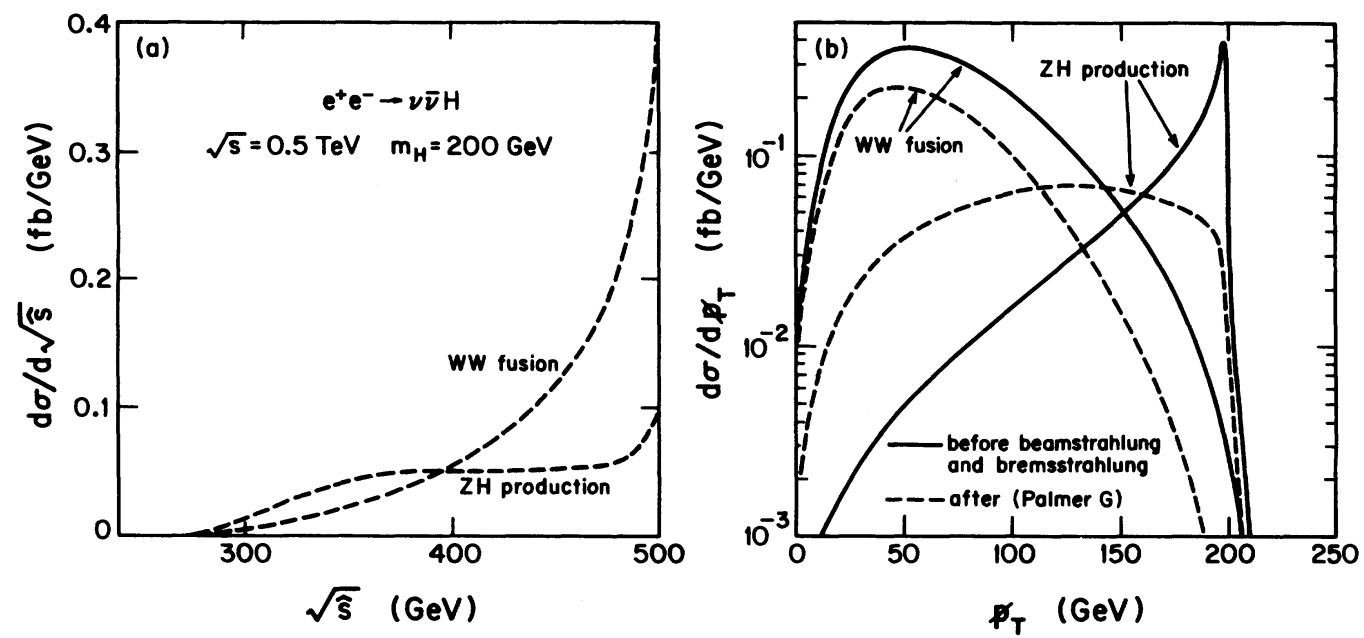

FIG. 11. Differential cross sections for $e^{+} e^{-} \rightarrow \nu \bar{\nu} H$, before and after bremsstrahlung and beamstrahlung (Palmer $G$ ) corrections: (a) $d \sigma / d \sqrt{\hat{s}}$ and (b) $d \sigma / d p_{T}$. Both $W W$-fusion and $Z H$-production channels are shown, assuming $m_{H}=200 \mathrm{GeV}$. Dashed (solid) curves denote results with (without) QED radiative corrections.

with a falling cross section, contributes across a wide range of $\hat{s}$. Figure 11(b) shows the $\not_{T}$ distributions before and after corrections; we see that contributions from the upper $\not_{T}$ range (that require close-to-maximum values of $\sqrt{\hat{s}}$ ) are strongly suppressed, and the Jacobian peak near maximum $\not_{T}$ is smeared out.

In Fig. 12 we investigate the impact of bremsstrahlung and beamstrahlung on $e^{+} e^{-} \rightarrow \nu \bar{\nu} W^{+} W^{-}$differential cross sections, distinguishing between the scattering and annihilation channels; this contains the Higgs-boson signals previously shown in Fig. 11, but now the intrinsic backgrounds are also present. Figures 12(a) and 12(d) display the $\sqrt{\hat{s}}$ dependences. Figures $12(\mathrm{~b})$ and 12(e) show the distributions versus invariant mass $m_{W W}$; ob-
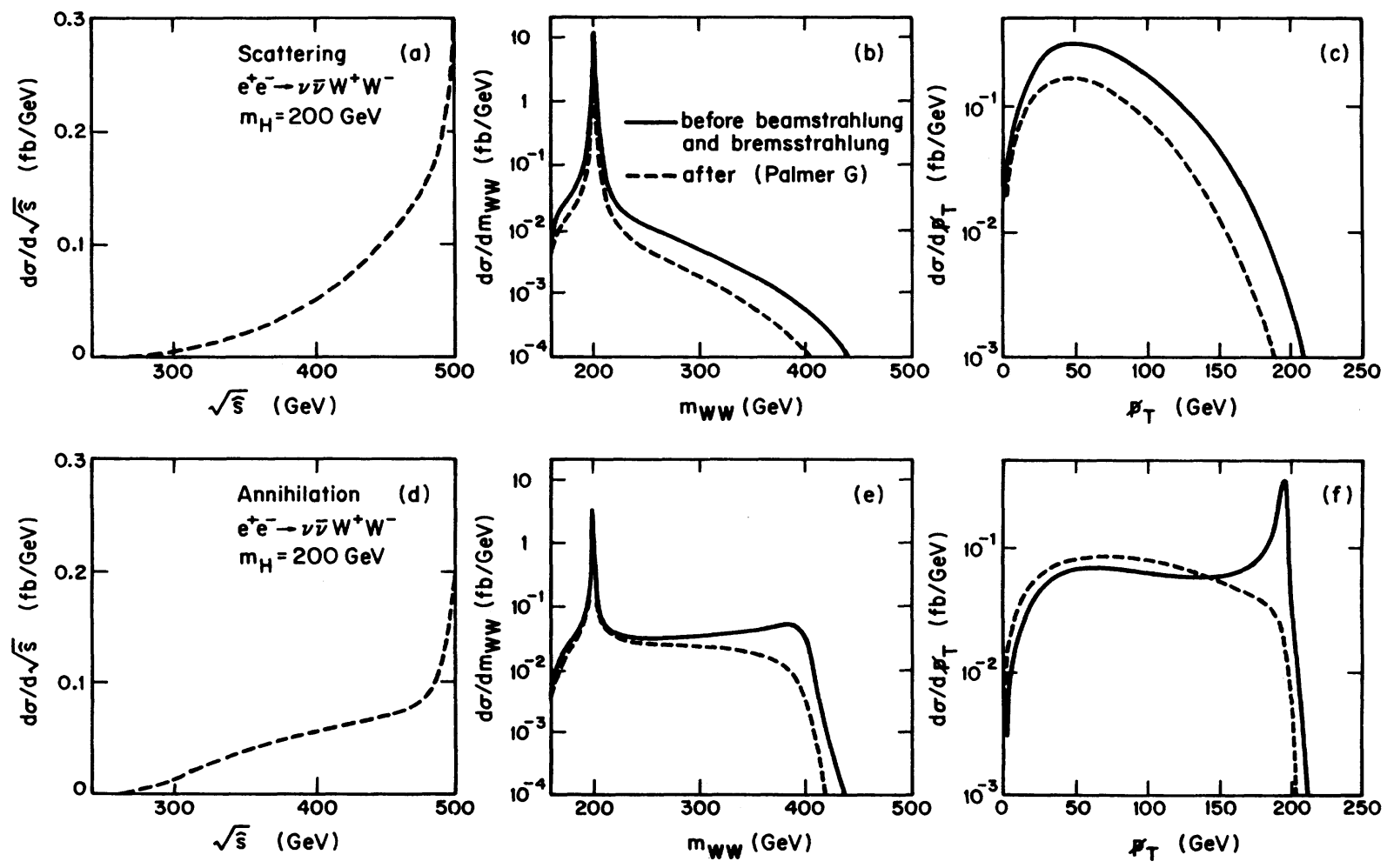

FIG. 12. Differential cross sections, before and after bremsstrahlung and beamstrahlung (Palmer $G$ ) corrections, for $e^{+} e^{-} \rightarrow$ $\nu \bar{\nu} W^{+} W^{-}$in the scattering channel, assuming $m_{H}=200 \mathrm{GeV}:$ (a) $d \sigma / d \sqrt{\hat{s}}$, (b) $d \sigma / d m_{V V}$, and (c) $d \sigma / d p_{T}$. The corresponding distributions for the annihilation channel are shown in (d), (e), and (f). Dashed (solid) curves denote results with (without) QED radiative corrections. 

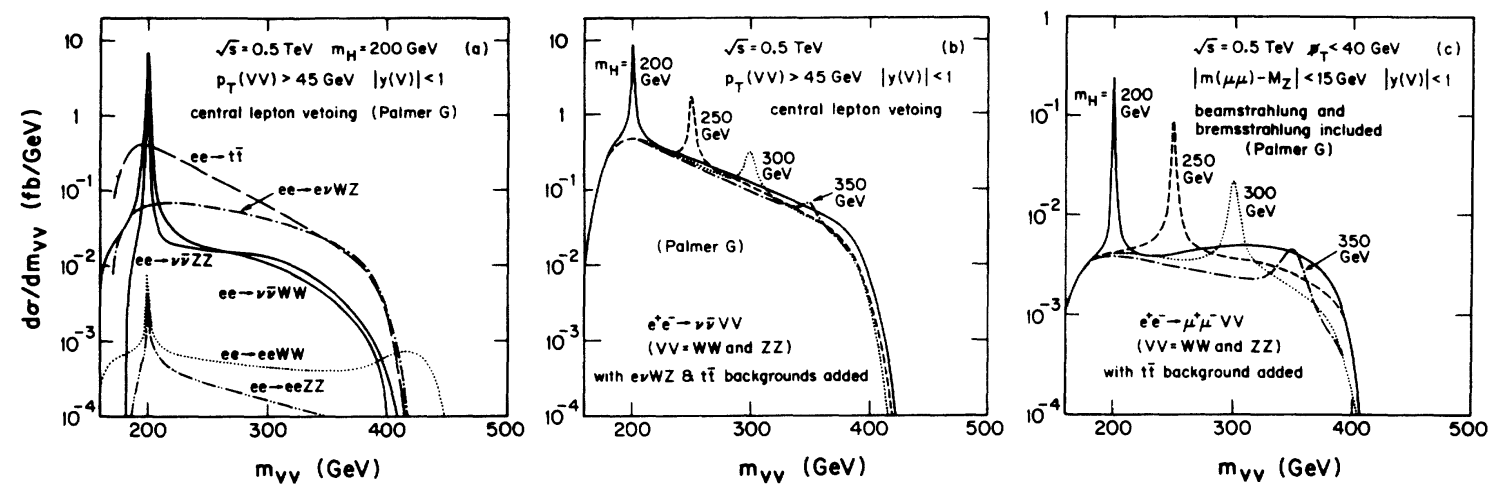

FIG. 13. Differential cross sections $d \sigma / d m_{V V}$ for heavy-Higgs signals and backgrounds, including all acceptance cuts plus bremsstrahlung and beamstrahlung corrections for the case of Palmer $G$ : (a) $\nu \bar{\nu} W W$ and $\nu \bar{\nu} Z Z$ signals and backgrounds separately for $m_{H}=200 \mathrm{GeV}$; (b) $\nu \bar{\nu} W W$ and $\nu \bar{\nu} Z Z$ channels, as well as the corresponding backgrounds from $e \nu W Z$ and $t \bar{t}$ are summed, for $m_{H}=200,250,300$, and $350 \mathrm{GeV}$; (c) $\mu \mu W W$ and $\mu \mu Z Z$ channels plus the corresponding $t \bar{t}$ background are summed, for $m_{H}=200,250,300$, and $350 \mathrm{GeV}$. Subsequent $V V \rightarrow j j j j$ decays may be included by multiplication with $[B(V \rightarrow j j)]^{2}=0.5$. The $t \bar{t}$ backgrounds here are for the case $m_{t}=150 \mathrm{GeV}$.
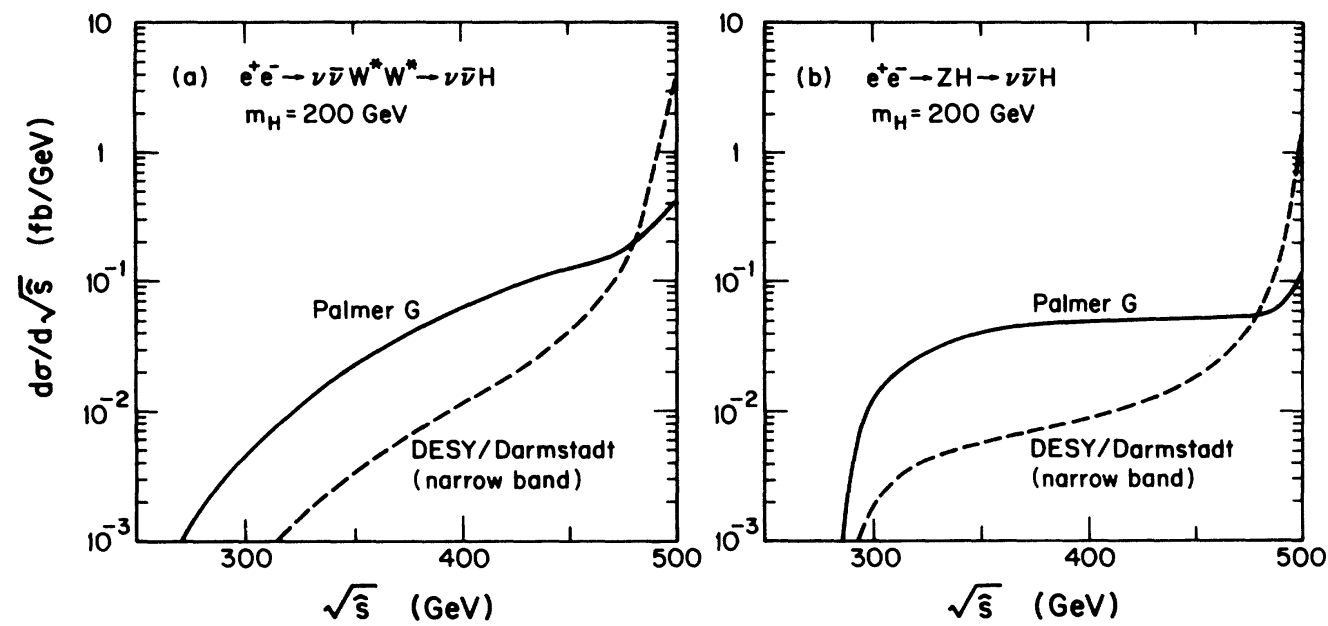

FIG. 14. Differential cross section $d \sigma / d \sqrt{\hat{s}}$ for $e^{+} e^{-} \rightarrow H \nu \bar{\nu}$, assuming $m_{H}=200 \mathrm{GeV}$, via (a) $W W$-fusion and (b) $Z H$ production. Beamstrahlung effects are shown for Palmer $G$ and DESY/Darmstadt (narrow band); bremsstrahlung is included also.
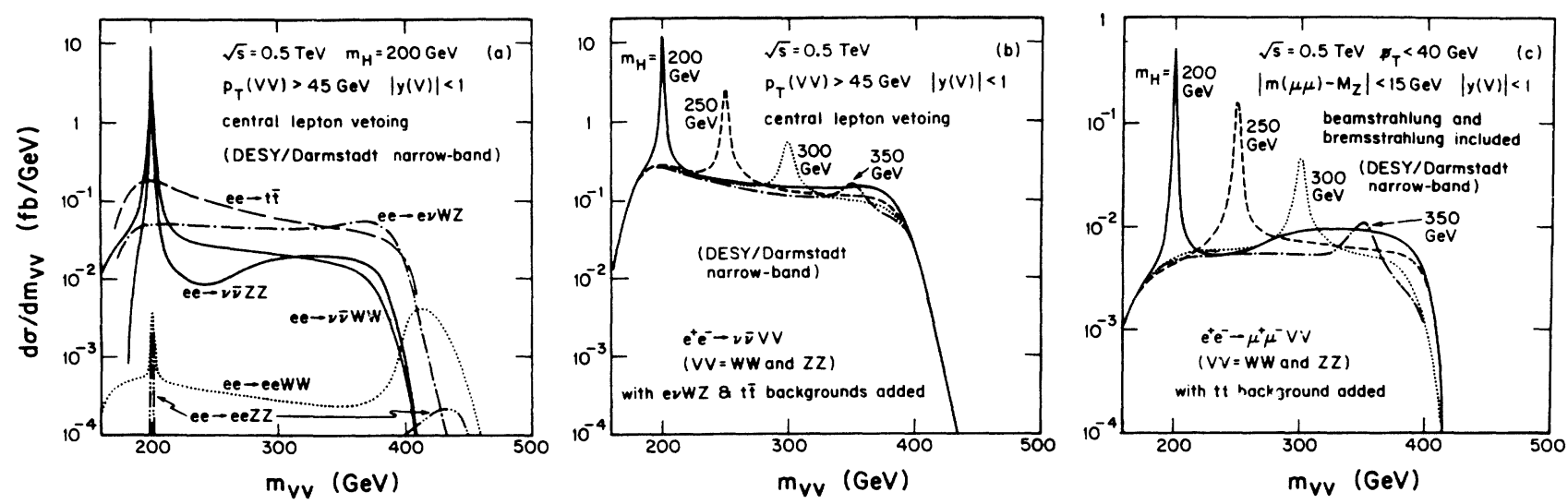

FIG. 15. Same as Fig. 13 but for the DESY/Darmstadt narrow-band design. 
viously, the smearing in $\hat{s}$ has no effect on locations and widths of the Higgs resonance peaks, but the height of each peak and the distribution of its intrinsic background can be changed. Figures 12(c) and 12(f) show the smearing of the $\not p_{T}$ distributions.

The lower entries in Tables I and II give the final cross sections for the signals and backgrounds in the $\nu \bar{\nu} V V$ and $\mu \mu V V$ channels, after adding bremsstrahlung and Palmer $G$-type beamstrahlung corrections to the previous results shown in the respective upper entries; we recall that this is the worst beamstrahlung scenario. In the $\nu \bar{\nu} V V$ channels, the Higgs signal is appreciably reduced while the background from $e \nu W Z$ is somewhat less reduced (or even increased). The $t \bar{t}$ background is doubled, partly because $t \bar{t}$ production is increased but mostly because the cuts become less effective at lower $\sqrt{\hat{s}}$. Nevertheless, a healthy signal remains right through the mass range illustrated here. For $m_{H}=300(350) \mathrm{GeV}$ the total signal would amount to $2.7(0.8) \mathrm{fb}$ compared to a total background of 40 (39) fb, of which the component under the mass peak would be only about $4(1.3) \mathrm{fb}$; see Fig. 13. If we assume as before an annual integrated luminosity of $50 \mathrm{fb}^{-1}$ and $50 \%$ instrumental efficiency and take dijet branching fractions $B(V \rightarrow j j)=0.7$, then for $m_{H}=300 \mathrm{GeV}$ there would be about 30 events/year in the combined $\nu \bar{\nu} V V \rightarrow \nu \bar{\nu} j j j j$ channels, with a background of about 50 events/year in the mass bin below the Higgs resonance peak. For $m_{H}=350 \mathrm{GeV}$ there would be about 10 signal events/year, with about 15 background events/year below the peak. It appears that a Higgs signal would be detectable in this channel for a mass up to $m_{H}=300 \mathrm{GeV}$ quite readily, and up to $m_{H}=350 \mathrm{GeV}$ eventually. The net Higgs-boson signal in the $\mu \mu V V$ channels is smaller by a factor of $15-40$, depending on $m_{H}$.

Figure 13 shows the total Higgs signals and backgrounds in the $\nu \bar{\nu} V V$ and $\mu \mu V V$ channels versus invariant mass $m_{V V}$. All the cuts (8)-(11) for $\nu \bar{\nu} V V$ and (7), (8), (9), (12) for $\mu \mu V V$ have been applied here, and bremsstrahlung and Palmer $G$ beamstrahlung corrections have been included. In Fig. 13(a) all the signals and backgrounds for $\nu \bar{\nu} V V$ channels are shown for $m_{H}=200 \mathrm{GeV}$. In Fig. 13(b) we show the sum of $\nu \bar{\nu} W W$ and $\nu \bar{\nu} Z Z$ differential cross sections, since these channels would be practically indistinguishable in their hadronic decay modes $V V \rightarrow j j j j$, for $m_{H}=200,250,300$, and $350 \mathrm{GeV}$. At the same time the major backgrounds from $e^{+} e^{-} \rightarrow e \nu W Z$ and from $e^{+} e^{-} \rightarrow t \bar{t}$, for the same choices of $m_{H}$, are added to the corresponding Higgs-boson signal. Similarly, Fig. 13(c) shows the combined differential cross sections of the $\mu \mu W W$ and $\mu \mu Z Z$ signals and the $t \bar{t}$ background for the same choices of $m_{H}$. This figure neatly summarizes our principal heavy-Higgs-boson results in the Palmer $G$ case.

We now examine the performances of the DESY/Darmstadt and TESLA designs with regard to beamstrahlung suppression. In Fig. 14 we compare for $m_{H}=200 \mathrm{GeV}$ the $\sqrt{\hat{s}}$ dependences of the differential cross sections $d \sigma / d \sqrt{\hat{s}}$ for $\nu \bar{\nu} H$ production which arise from Palmer $G$ and DESY/Darmstadt (narrow band). We consider separately the contributions from $W W$ fu- sion in Fig. 14(a) and from $Z H$ associated production in Fig. 14(b). The curves for Palmer $G$ are the same as in Fig. 11(a). We see that the distributions for DESY/Darmstadt are much more massed close to the nominal value $\sqrt{s}=500 \mathrm{GeV}$, i.e., the average energy loss of the incident beams by beamstrahlung is drastically reduced. In particular, the plateau in the curve of $Z H$ production is absent for this design.

The parenthesized numbers in the tables have been obtained by adopting the DESY/Darmstadt design parameters; the TESLA design gives almost identical results. The results lie throughout much closer to the uncorrected case than to the case of Palmer $G$. We conclude that the smearing effects of beamstrahlung do not necessarily represent a serious danger for Higgs hunting at a $500 \mathrm{GeV}$ NLC. It is an issue of machine architecture and operation to reduce unwanted beamstrahlung to the level of (unavoidable) bremsstrahlung.

Finally, in Fig. 15 we repeat the analysis of Fig. 13 for the DESY/Darmstadt design. The $m_{V V}$ distributions shown in Fig. 15(a) are much closer to the uncorrected case of Fig. 8 than to the Palmer $G$ case of Fig. 13(a). In particular, the bumps at the upper end of the $m_{V V}$ range in the distributions of the eeVV $(V V=W W, Z Z)$ channels, which can be traced to those annihilation diagrams where the $V$ bosons are emitted from the initial state [see Figs. 4(x) and 4(y)], are much less washed out here than in the case of Palmer $G$. Furthermore, the signal peaks are more prominent than for Palmer $G$; however, as we have noted already in the context of Figs. 12(b) and 12(d), their locations and widths are insensitive to beamstrahlung. These observations are substantiated by Figs. 15(b) and 15(c). The signals are somewhat bigger and the backgrounds are somewhat smaller than for the Palmer $G$ design; we conclude once more that a Higgs signal would be detectable in the $\nu \bar{\nu} V V$ channel up to $m_{H}=350 \mathrm{GeV}$.

\section{SUMMARY}

We have studied standard-model Higgs-boson signals at a possible future $e^{+} e^{-}$collider with c.m. energy $\sqrt{s}=$ $0.5 \mathrm{TeV}$. Our results may be summarized as follows.

(i) For an intermediate-mass Higgs boson in the range $M_{Z}<m_{H}<2 M_{W}$, the $Z H$-production channel offers the biggest production cross section, even below the nominal $Z H$ threshold; see Fig. 2. We have updated the branching fractions into different decay modes, which determine the detectability, for this mass range; see Fig. 3.

(ii) For a heavy Higgs boson, with $m_{H}>2 M_{W}$, the most promising signals are in the channels $e^{+} e^{-} \rightarrow$ $\nu \bar{\nu} H \rightarrow \nu \bar{\nu} V V$, where $V=W, Z$ and $V \rightarrow j j$ dijet decays are detected. Various backgrounds

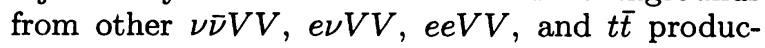
tion mechanisms can be greatly suppressed by a $V$-rapidity cut, a missing transverse momentum $\left[=p_{T}(V V)\right]$ cut, and a central-lepton veto. A detectable $\nu \bar{\nu} j j j j$ signal is predicted up to masses of order $m_{H}=300-350 \mathrm{GeV}$; see Table I and 
Figs. 13(b) and 15(b). Assuming an annual integrated luminosity of $50 \mathrm{fb}^{-1}, 50 \%$ instrumental efficiency, and a pessimistic beamstrahlung scenario, a mass value $m_{H}=300$ (350) $\mathrm{GeV}$ would imply about 30 (10) signal events per year in a narrow $m(j j j j)$ peak, with about 50 (15) background events under this peak.

(iii) Another, smaller, Higgs-boson signal appears in the channel $e^{+} e^{-} \rightarrow Z H \rightarrow \mu^{+} \mu^{-} V V$. This is not threatened by large backgrounds and requires no stringent cuts. For the same assumed luminosity and efficiency as above a $\mu \mu j j j j$ signal of about 5 (3) events/year above a relatively small background may be expected for $m_{H}=250$ (300) GeV; see Table II and Figs. 13(c) and 15(c). This signal arises entirely from the $Z H$ diagram. The cross section for this signal is a factor of 10-40 times smaller than that in the $\nu \bar{\nu} V V$ channels.

(iv) A third Higgs-boson signal appears in the channel $e^{+} e^{-} \rightarrow Z H \rightarrow 6$ jets. The signal here is comparable in size with the $\nu \bar{\nu} j j j j$ signal, but the background is much larger; precise predictions would require detailed jet simulation studies.

(v) We have used minimal illustrative cuts to bring the backgrounds under control. Further suppression of the $t \bar{t}$ backgrounds can in principle be achieved by vetoing $\nu \bar{\nu} V V$ candidate events where $m(j j j) \approx$ $m_{t}$, and $\mu \mu V V$ candidate events where one muon is not isolated. The $m(j j j)$ cut can however be damaging to the Higgs-boson signal, depending on $m_{t}$ and $m_{H}$.

(vi) The $\nu \bar{\nu} H$ signal receives contributions both from $Z H$-production and from $W W$-fusion mechanisms. These contributions can in principle be separated on the basis of their different $p_{T}(V V)$ dependences in the $\nu \bar{\nu} V V$ channel, or by comparing the $\nu \bar{\nu} V V$ and $\mu \mu V V$ signals. If these contributions are measured separately, they will allow a direct comparison of the $Z Z H$ and $W W H$ coupling strengths.

(vii) Bremsstrahlung and beamstrahlung corrections are very important. They lower the subprocess c.m. energy and momentum, enhancing annihilation channels and suppressing scattering channels of Higgs-boson production, and smearing out some kinematical features such as Jacobian peaks in $p_{T}(V V)$. Our calculations of heavy-Higgs-boson signals include both initial-state bremsstrahlung effects and illustrative calculations of beamstrahlung. The net effect in our present cases is to reduce signals and increase backgrounds.

(viii) The measurability of heavy-Higgs-boson signals at an $e^{+} e^{-}$collider with $\sqrt{s}=0.5 \mathrm{TeV}$ is illustrated most dramatically in Figs. 13 and 15. Here all major backgrounds and all our selection cuts plus bremsstrahlung and illustrative beamstrahlung effects are fully included; experimental smearing in the $H \rightarrow V V$ invariant mass distribution is still to be added.

\section{ACKNOWLEDGMENTS}

We thank Tim Barklow for making available to us his beamstrahlung simulation program, Kaoru Hagiwara for a clarifying comment regarding the treatment of $t$ channel photon exchanges in Ref. [3], Tao Han for valuable suggestions during the initial stages of this work, San Fu Tuan for encouraging us to complete this analysis, and Peter Zerwas for proposing a comparative study of the beamstrahlung performances of the various NLC designs. This work was supported in part by the U.S. Department of Energy under Contract No. DE-AC02-76ER00881 and in part by the University of Wisconsin Research Committee with funds granted by the Wisconsin Alumni Research Foundation.

\section{APPENDIX A: FEYNMAN AMPLITUDES}

In this appendix we list the matrix elements for all the considered processes, from which explicit helicity amplitudes can be directly computed. To start with, we introduce some general notation:

$$
\begin{aligned}
g_{a}^{W}(f) & =-g_{b}^{W}(f)=\frac{g}{2 \sqrt{2}}, \\
g_{a}^{Z}(f) & =g_{Z}\left(\frac{T_{3 f}}{2}-Q_{f} x_{W}\right) \\
g_{b}^{Z}(f) & =-g_{Z} \frac{T_{3 f}}{2} \\
g_{a}^{\gamma}(f) & =e Q_{f} \\
g_{b}^{\gamma}(f) & =0 \\
g^{V}(f) & =g_{a}^{V}(f)+g_{b}^{V}(f) \gamma^{5} \\
D^{X}(k) & =\frac{1}{k^{2}-M_{X}^{2}+i \Gamma_{X}\left(k^{2}\right) M_{X}}, \quad(V=\gamma, W, Z), \quad \Gamma_{X}\left(k^{2}\right)=\Gamma_{X} \theta\left(k^{2}\right) \quad(\text { with } X=\gamma, W, Z, H) \\
P_{V}^{\alpha \beta}(k) & =\left[g^{\alpha \beta}+\frac{(1-\xi) k^{\alpha} k^{\beta}}{\xi k^{2}-M_{V}^{2}}\right] D^{V}(k),
\end{aligned}
$$




$$
\begin{aligned}
\Gamma^{\alpha}\left(k_{1}, k_{2} ; \epsilon_{1}, \epsilon_{2}\right) & =\left(k_{1}-k_{2}\right)^{\alpha} \epsilon_{1} \cdot \epsilon_{2}+\left(2 k_{2}+k_{1}\right) \cdot \epsilon_{1} \epsilon_{2}^{\alpha}-\left(2 k_{1}+k_{2}\right) \cdot \epsilon_{2} \epsilon_{1}^{\alpha}, \\
g_{V W W} & = \begin{cases}e \cot \theta_{W} & \text { for } V=Z \\
e & \text { for } V=\gamma\end{cases}
\end{aligned}
$$

Here $Q_{f}$ and $T_{3 f}$ are the electric charge (in units of the positron charge) and the third component of weak isospin of the fermion $f, g$ is the $\mathrm{SU}(2)$ gauge coupling, and $g_{Z}=g / \cos \theta_{W}, x_{W}=\sin ^{2} \theta_{W}$, with $\theta_{W}$ being the weak mixing angle in the standard model. Dots between four-vectors denote scalar products and $g_{\alpha \beta}$ is the Minkowskian metric tensor with $g_{00}=-g_{11}=-g_{22}=$ $-g_{33}=1 ; \xi$ is a gauge-fixing parameter.

In Fig. $4, p_{i}(i=1, \ldots, 4)$ denote the momenta flowing along the corresponding fermion lines in the direction of the arrows. We shall always denote the associated spinors by the symbols $u\left(p_{i}\right)$ and $\bar{u}\left(p_{i}\right)$ for the ingoing and outgoing arrows, which is usual for the annihilation and creation of fermions, respectively. When an ingoing arrow represents the production of an antifermion with physical momentum $p_{i}$ (momentum label $-p_{i}$ ), the symbol $u\left(-p_{i}\right)$ is defined to mean $v\left(p_{i}\right)$. A similar convention applies to fermion (antifermion) spins, although the spin labels are not written explicitly below. The symbols $\chi^{ \pm}$ in Figs. 4(e) and 4(g) denote the Goldstone bosons in $R_{\xi}$ gauge.

Finally, when adding the contributions from annihilation and scattering together, a relative sign change has to be introduced between the two sets of formulas given below. The spin labels of the spinors $u$ and $\bar{u}$, and the polarization labels of the $\epsilon$ are not shown explicitly. To obtain the final cross section, we sum over all the final fermion (antifermion) spins and weak-boson polarizations, and average the initial fermion (antifermion) spins.
1. $e^{+} e^{-} \rightarrow \nu \bar{\nu} W^{+} W^{-}$lepton scattering contributions

Lepton scattering comes from the generic diagrams depicted in Figs. 4(a)-(d), (f), (h)-(o), (p), (q), (s), (t), and (v) $-(\mathrm{y})$ with the substitutions

$$
\begin{aligned}
1 & \rightarrow e^{-}, & 2 & \rightarrow \nu_{e}, \\
3 & \rightarrow \nu_{e}, & 4 & \rightarrow e^{-}, \\
V_{1} & \rightarrow W^{-}, & V_{2} & \rightarrow W^{+} .
\end{aligned}
$$

In this channel fermion lines 1 and 4 represent the incoming particles. It is convenient to introduce the following shorthand notation:

$$
\begin{aligned}
u_{i} & =u\left(p_{i}\right), \quad \bar{u}_{i}=\bar{u}\left(p_{i}\right), \quad \epsilon_{i}=\epsilon\left(k_{i}\right), \\
J_{1}^{\alpha} & =\bar{u}_{2} \gamma^{\alpha} g^{W}(e) u_{1} D^{W}\left(p_{1}-p_{2}\right), \\
J_{2}^{\alpha} & =\bar{u}_{4} \gamma^{\alpha} g^{W}(e) u_{3} D^{W}\left(p_{3}-p_{4}\right), \\
\bar{u}_{i j}^{(m)} & =\bar{u}_{i} \xi_{j} g^{W}(e) \frac{\not p_{i}+\not k_{j}+m}{\left(p_{i}+k_{j}\right)^{2}-m^{2}}, \\
u_{j i}^{(m)} & =\frac{\not p_{i}-\not k_{j}+m}{\left(p_{i}-k_{j}\right)^{2}-m^{2}} k_{j} g^{W}(e) u_{i}, \\
\bar{u}_{2 i j}^{\left(m, m^{\prime}\right)} & =\bar{u}_{2 i}^{(m)} \xi_{j} g^{W}(e) \frac{\not p_{2}+\not k_{i}+\not k_{j}+m^{\prime}}{\left(p_{2}+k_{i}+k_{j}\right)^{2}-m^{\prime 2}}, \\
u_{j i 1}^{\left(m^{\prime}, m\right)} & =\frac{\not p_{1}-\not k_{i}-\not k_{j}+m^{\prime}}{\left(p_{1}-k_{i}-k_{j}\right)^{2}-m^{\prime 2}} k_{j} g^{W}(e) u_{i 1}^{(m)},
\end{aligned}
$$

where $m, m^{\prime}$ denote the (finite) electron mass or the (zero) neutrino mass in fermion propagators. Then the amplitudes read

$$
\begin{aligned}
\mathcal{M}^{(a)} & =-g^{2} M_{W}^{2} D^{H}\left(k_{1}+k_{2}\right) J_{1} \cdot J_{2} \epsilon_{1} \cdot \epsilon_{2} \\
\mathcal{M}^{(b)} & =-g^{2} M_{W}^{2} D^{H}\left(p_{1}-p_{2}-k_{1}\right) J_{1} \cdot \epsilon_{1} J_{2} \cdot \epsilon_{2} \\
\mathcal{M}^{(c)} & =g^{2}\left[2 J_{2} \cdot \epsilon_{1} J_{1} \cdot \epsilon_{2}-J_{2} \cdot \epsilon_{2} J_{1} \cdot \epsilon_{1}-J_{2} \cdot J_{1} \epsilon_{1} \cdot \epsilon_{2}\right] \\
\mathcal{M}^{(d)}= & \sum_{V=\gamma, Z} g_{V W W}^{2} P_{V}^{\alpha \beta}\left(k_{1}+k_{2}\right) \Gamma_{\alpha}\left(p_{1}-p_{2}, p_{3}-p_{4} ; J_{1}, J_{2}\right) \Gamma_{\beta}\left(k_{1}, k_{2} ; \epsilon_{1}, \epsilon_{2}\right) \\
\mathcal{M}^{(f)}= & \sum_{V=\gamma, Z} g_{V W W}^{2} P_{V}^{\alpha \beta}\left(p_{1}-p_{2}-k_{1}\right) \Gamma_{\alpha}\left(p_{1}-p_{2},-k_{1} ; J_{1}, \epsilon_{1}\right) \Gamma_{\beta}\left(-k_{2}, p_{3}-p_{4} ; \epsilon_{2}, J_{2}\right) \\
\mathcal{M}^{(h-k)}= & \sum_{V=\gamma, Z} P_{V}^{\alpha \beta}\left(p_{1}-p_{2}-k_{1}\right)\left[\bar{u}_{21}^{(m)} \gamma_{\alpha} g^{V}(e) u_{1}+\bar{u}_{2} \gamma_{\alpha} g^{V}(\nu) u_{11}^{(0)}\right] \\
& \times\left[\bar{u}_{42}^{(0)} \gamma_{\beta} g^{V}(\nu) u_{3}+\bar{u}_{4} \gamma_{\beta} g^{V}(e) u_{23}^{(m)}\right] \\
\mathcal{M}^{(l, m)}= & \sum_{V=\gamma, Z}-g_{V W W} P_{V}^{\alpha \beta}\left(p_{1}-p_{2}-k_{1}\right) \Gamma_{\alpha}\left(p_{1}-p_{2},-k_{1} ; J_{1}, \epsilon_{1}\right) \\
& \quad \times\left[\bar{u}_{42}^{(0)} \gamma_{\beta} g^{V}(\nu) u_{3}+\bar{u}_{4} \gamma_{\beta} g^{V}(e) u_{23}^{(m)}\right] \\
\mathcal{M}^{(n, o)}= & \sum_{V=\gamma, Z}-g_{V W W} P_{V}^{\alpha \beta}\left(p_{1}-p_{2}-k_{1}\right) \Gamma_{\alpha}\left(-k_{2}, p_{3}-p_{4} ; \epsilon_{2}, J_{2}\right) \\
\quad & \quad\left[\bar{u}_{21}^{(m)} \gamma_{\beta} g^{V}(e) u_{1}+\bar{u}_{2} \gamma_{\beta} g^{V}(\nu) u_{11}^{(0)}\right]
\end{aligned}
$$




$$
\begin{aligned}
\mathcal{M}^{(p, q)} & =\bar{u}_{212}^{(m, 0)} J_{2} g^{W}(e) u_{1}+\bar{u}_{2} J_{2} g^{W}(e) u_{211}^{(m, 0)} \\
\mathcal{M}^{(s, t)} & =\bar{u}_{421}^{(0, m)} J_{1} g^{W}(e) u_{3}+\bar{u}_{4} J_{1} g^{W}(e) u_{123}^{(0, m)}, \\
\mathcal{M}^{(v)} & =\sum_{V=\gamma, Z}-g_{V W W} D^{V}\left(k_{1}+k_{2}\right) \bar{u}_{4} \mp\left(k_{1}, k_{2} ; \epsilon_{1}, \epsilon_{2}\right) g^{V}(e) \frac{\not p_{4}+\not k_{1}+\not k_{2}+m}{\left(p_{4}+k_{1}+k_{2}\right)^{2}-m^{2}} J_{1} g^{W}(e) u_{3}, \\
\mathcal{M}^{(w)} & =-g_{Z W W} D^{Z}\left(k_{1}+k_{2}\right) \bar{u}_{4} J_{1} g^{W}(e) \frac{\not p_{3}-\not k_{1}-\not k_{2}}{\left(p_{3}-k_{1}-k_{2}\right)^{2}} \mp\left(k_{1}, k_{2} ; \epsilon_{1}, \epsilon_{2}\right) g^{Z}(\nu) u_{3}, \\
\mathcal{M}^{(x)} & =\sum_{V=\gamma, Z}-g_{V W W} D^{V}\left(k_{1}+k_{2}\right) \bar{u}_{2} J_{2} g^{W}(e) \frac{\not p_{1}-\not k_{1}-\not k_{2}+m}{\left(p_{1}-k_{1}-k_{2}\right)^{2}-m^{2}} \not\left(k_{1}, k_{2} ; \epsilon_{1}, \epsilon_{2}\right) g^{V}(e) u_{1}, \\
\mathcal{M}^{(y)} & =-g_{Z W W} D^{Z}\left(k_{1}+k_{2}\right) \bar{u}_{2} \mp\left(k_{1}, k_{2} ; \epsilon_{1}, \epsilon_{2}\right) g^{Z}(\nu) \frac{\not p_{2}+\not k_{1}+\not k_{2}}{\left(p_{2}+k_{1}+k_{2}\right)^{2}} J_{2} g^{W}(e) u_{1} .
\end{aligned}
$$

\section{2. $e^{+} e^{-} \rightarrow \nu \bar{\nu} W^{+} W^{-}$lepton annihilation contributions}

Lepton annihilation contributes via the generic diagrams depicted in Figs. 4(a), (c), (f), (g), (h), (j), and (l)-(y) with the substitutions

$$
\begin{aligned}
1 & \rightarrow e^{-}, & 2 & \rightarrow e^{-}, \\
3 & \rightarrow \nu, & 4 & \rightarrow \nu, \\
V_{1} & \rightarrow W^{-}, & V_{2} & \rightarrow W^{+} .
\end{aligned}
$$

Here fermion lines 1 and 2 represent the incoming particles. Redefining

$$
\begin{aligned}
& J_{1 \alpha}^{V}=\bar{u}_{2} \gamma_{\alpha} g^{V}(e) u_{1} D^{V}\left(p_{1}-p_{2}\right), \\
& J_{2 \alpha}=\bar{u}_{4} \gamma_{\alpha} g^{Z}(\nu) u_{3} D^{Z}\left(p_{3}-p_{4}\right),
\end{aligned}
$$

we find the amplitudes

$$
\begin{aligned}
\mathcal{M}^{(a)}= & -\frac{g^{2}}{1-x_{W}} M_{W}^{2} D^{H}\left(k_{1}+k_{2}\right) \epsilon_{1} \cdot \epsilon_{2} J_{1}^{Z} \cdot J_{2}, \\
\mathcal{M}^{(c)}= & \sum_{V=\gamma, Z}-g_{V W W} g_{Z W W}\left[2 \epsilon_{1} \cdot \epsilon_{2} J_{1}^{V} \cdot J_{2}-\epsilon_{1} \cdot J_{1}^{V} \epsilon_{2} \cdot J_{2}-\epsilon_{1} \cdot J_{2} \epsilon_{2} \cdot J_{1}^{V}\right] \\
\mathcal{M}^{(f)}= & \sum_{V=\gamma, Z}\left[g_{V W W} g_{Z W W} P_{W}^{\alpha \beta}\left(p_{1}-p_{2}-k_{1}\right) \Gamma_{\alpha}\left(-k_{1}, p_{1}-p_{2} ; \epsilon_{1}, J_{1}^{V}\right) \Gamma_{\beta}\left(p_{3}-p_{4},-k_{2} ; J_{2}, \epsilon_{2}\right)\right. \\
& \left.\quad+g_{V W W} g_{Z W W} P_{W}^{\alpha \beta}\left(p_{1}-p_{2}-k_{2}\right) \Gamma_{\alpha}\left(p_{1}-p_{2},-k_{2} ; J_{1}^{V}, \epsilon_{2}\right) \Gamma_{\beta}\left(-k_{1}, p_{3}-p_{4} ; \epsilon_{1}, J_{2}\right)\right] \\
\mathcal{M}^{(g)}= & \sum_{V=\gamma, Z} g^{2} x_{W} M_{W}^{2} J_{1}^{V} \cdot \epsilon_{1} J_{2} \cdot \epsilon_{2} \frac{\xi}{\xi\left(p_{1}-p_{2}-k_{1}\right)^{2}-M_{W}^{2}} \\
& \quad \times\left\{\begin{array}{cc}
-\tan ^{2} \theta_{W} & \text { if } V=Z \\
\tan \theta_{W} & \text { if } V=\gamma
\end{array}\right\}+\left(k_{1} \leftrightarrow k_{2}\right), \\
\mathcal{M}^{(h)}= & P_{W}^{\alpha \beta}\left(p_{1}-p_{2}-k_{2}\right) \bar{u}_{22}^{(0)} \gamma_{\alpha} g^{W}(e) u_{1} \bar{u}_{41}^{(m)} \gamma_{\beta} g^{W}(e) u_{3}, \\
\mathcal{M}^{(j)}= & P_{W}^{\alpha \beta}\left(p_{1}-p_{2}-k_{1}\right) \bar{u}_{2} \gamma_{\alpha} g^{W}(e) u_{11}^{(0)} \bar{u}_{4} \gamma_{\beta} g^{W}(e) u_{23}^{(m)}, \\
\mathcal{M}^{(l)}= & \sum_{V=\gamma, Z}-g_{V W W} P_{W}^{\alpha \beta}\left(p_{1}-p_{2}-k_{2}\right) \Gamma_{\alpha}\left(p_{1}-p_{2},-k_{2} ; J_{1}^{V}, \epsilon_{2}\right) \bar{u}_{41}^{(m)} \gamma_{\beta} g^{W}(e) u_{3}, \\
\mathcal{M}^{(m)}= & \sum_{V=\gamma, Z}-g_{V W W} P_{W}^{\alpha \beta}\left(p_{1}-p_{2}-k_{1}\right) \Gamma_{\alpha}\left(-k_{1}, p_{1}-p_{2} ; \epsilon_{1}, J_{1}^{V}\right) \bar{u}_{4} \gamma_{\beta} g^{W}(e) u_{23}^{(m)}, \\
\mathcal{M}^{(n)}= & -g_{Z W W} P_{W}^{\alpha \beta}\left(p_{1}-p_{2}-k_{2}\right) \Gamma_{\alpha}\left(-k_{1}, p_{3}-p_{4} ; \epsilon_{1}, J_{2}\right) \bar{u}_{22}^{(0)} \gamma_{\beta} g^{W}(e) u_{1}, \\
\mathcal{M}^{(o)}= & -g_{Z W W} P_{W}^{\alpha \beta}\left(p_{1}-p_{2}-k_{1}\right) \Gamma_{\alpha}\left(p_{3}-p_{4},-k_{2} ; J_{2}, \epsilon_{2}\right) \bar{u}_{2} \gamma_{\beta} g^{W}(e) u_{11}^{(0)}, \\
\mathcal{M}^{(p-r)}= & \bar{u}_{22}^{(0)} J_{2} g^{Z}(\nu) u_{11}^{(0)}+\bar{u}_{2} J_{2} g^{Z}(e) u_{211}^{(m, 0)}+\bar{u}_{221}^{(0, m)} J_{2} g^{Z}(e) u_{1},
\end{aligned}
$$




$$
\begin{aligned}
\mathcal{M}^{(s-u)} & =\bar{u}_{4} J_{1}^{Z} g^{Z}(\nu) u_{123}^{(0, m)}+\sum_{V=\gamma, Z} \bar{u}_{41}^{(m)} J_{1}^{V} g^{V}(e) u_{23}^{(m)}+\bar{u}_{412}^{(m, 0)} J_{1}^{Z} g^{Z}(\nu) u_{3}, \\
\mathcal{M}^{(v)} & =-g_{Z W W} D^{Z}\left(k_{1}+k_{2}\right) \bar{u}_{4} \Psi\left(k_{1}, k_{2} ; \epsilon_{1}, \epsilon_{2}\right) g^{Z}(\nu) \frac{\not p_{4}+\not k_{1}+\not k_{2}}{\left(p_{4}+k_{1}+k_{2}\right)^{2}} J_{1}^{Z} g^{Z}(\nu) u_{3}, \\
\mathcal{M}^{(w)} & =-g_{Z W W} D^{Z}\left(k_{1}+k_{2}\right) \bar{u}_{4} J_{1}^{Z} g^{Z}(\nu) \frac{\not p_{3}-\not k_{1}-\not k_{2}}{\left(p_{3}-k_{1}-k_{2}\right)^{2}} \not\left(k_{1}, k_{2} ; \epsilon_{1}, \epsilon_{2}\right) g^{Z}(\nu) u_{3}, \\
\mathcal{M}^{(x)} & =\sum_{V=\gamma, Z}-g_{V W W} D^{V}\left(k_{1}+k_{2}\right) \bar{u}_{2} J_{2} g^{Z}(e) \frac{\not p_{1}-\not k_{1}-\not k_{2}+m}{\left(p_{1}-k_{1}-k_{2}\right)^{2}-m^{2}} \not\left(k_{1}, k_{2} ; \epsilon_{1}, \epsilon_{2}\right) g^{V}(e) u_{1}, \\
\mathcal{M}^{(y)} & =\sum_{V=\gamma, Z}-g_{V W W} D^{V}\left(k_{1}+k_{2}\right) \bar{u}_{2} \not\left(k_{1}, k_{2} ; \epsilon_{1}, \epsilon_{2}\right) g^{V}(e) \frac{\not p_{2}+\not k_{1}+\not k_{2}+m}{\left(p_{2}+k_{1}+k_{2}\right)^{2}-m^{2}} J_{2} g^{Z}(e) u_{1} .
\end{aligned}
$$

\section{3. $e^{+} e^{-} \rightarrow \nu \bar{\nu} Z Z$ lepton scattering contributions}

Lepton scattering comes from the generic diagrams depicted in Figs. 4(a), (c), and (f)-(u) with the substitutions

$$
\begin{aligned}
1 & \rightarrow e^{-}, & 2 & \rightarrow \nu_{e}, \\
3 & \rightarrow \nu_{e}, & 4 & \rightarrow e^{-}, \\
V_{1} & \rightarrow Z, & V_{2} & \rightarrow Z .
\end{aligned}
$$

Here fermion lines 1 and 4 represent the incoming particles. Redefining

$$
\begin{aligned}
J_{1 \alpha} & =\bar{u}_{2} \gamma_{\alpha} g^{W}(e) u_{1} D^{W}\left(p_{1}-p_{2}\right), \\
J_{2 \alpha} & =\bar{u}_{4} \gamma_{\alpha} g^{W}(e) u_{3} D^{W}\left(p_{3}-p_{4}\right), \\
u_{j i, f}^{(m)} & =\frac{\not p_{i}-\not k_{j}+m}{\left(p_{i}-k_{j}\right)^{2}-m^{2}} \xi_{j} g^{Z}(f) u_{i}, \\
\bar{u}_{i j, f}^{(m)} & =\bar{u}_{i} \xi_{j} g^{Z}(f) \frac{\not p_{i}+\not k_{j}+m}{\left(p_{i}+k_{j}\right)^{2}-m^{2}}, \\
\bar{u}_{2 i j, f_{1} f_{2}}^{\left(m, m^{\prime}\right)} & =\bar{u}_{2 i, f_{1}}^{(m)} \xi_{j} g^{Z}\left(f_{2}\right) \frac{\not p_{2}+\not k_{i}+\not k_{j}+m^{\prime}}{\left(p_{2}+k_{i}+k_{j}\right)^{2}-m^{2}}, \\
u_{j i 1, f_{2} f_{1}}^{\left(m^{\prime}, m\right)} & =\frac{\not p_{1}-\not k_{i}-\not k_{j}+m^{\prime}}{\left(p_{1}-k_{i}-k_{j}\right)^{2}-m^{\prime 2}} \xi_{j} g^{Z}\left(f_{2}\right) u_{i 1, f_{1}}^{(m)},
\end{aligned}
$$

where $f, f_{1}$, and $f_{2}$ in the subscript denote the fermions to which the external $Z$ bosons are attached. We find the amplitudes

$$
\begin{aligned}
\mathcal{M}^{(a)}= & -\frac{g^{2}}{1-x_{W}} M_{W}^{2} D^{H}\left(k_{1}+k_{2}\right) \epsilon_{1} \cdot \epsilon_{2} J_{1} \cdot J_{2} \\
\mathcal{M}^{(c)}= & -g^{2}\left(1-x_{W}\right)\left[2 \epsilon_{1} \cdot \epsilon_{2} J_{1} \cdot J_{2}-\epsilon_{1} \cdot J_{1} \epsilon_{2} \cdot J_{2}-\epsilon_{1} \cdot J_{2} \epsilon_{2} \cdot J_{1}\right] \\
\mathcal{M}^{(f)}= & g_{Z W W}^{2} P_{W}^{\alpha \beta}\left(p_{1}-p_{2}-k_{1}\right) \Gamma_{\alpha}\left(-k_{1}, p_{1}-p_{2} ; \epsilon_{1}, J_{1}\right) \Gamma_{\beta}\left(p_{3}-p_{4},-k_{2} ; J_{2}, \epsilon_{2}\right) \\
\mathcal{M}^{(g)}= & -\frac{g^{2}}{1-x_{W}} x_{W}^{2} M_{W}^{2} \epsilon_{1} \cdot J_{1} \epsilon_{2} \cdot J_{2} \frac{\xi}{\xi\left(p_{1}-p_{2}-k_{1}\right)^{2}-M_{W}^{2}} \\
\mathcal{M}^{(h-k)}= & P_{W}^{\alpha \beta}\left(p_{1}-p_{2}-k_{1}\right)\left[\bar{u}_{2} \gamma_{\alpha} g^{W}(e) u_{11, e}^{(m)}+\bar{u}_{21, \nu}^{(0)} \gamma_{\alpha} g^{W}(e) u_{1}\right] \\
& \times\left[\bar{u}_{4} \gamma_{\beta} g^{W}(e) u_{23, \nu}^{(0)}+\bar{u}_{42, e}^{(m)} \gamma_{\beta} g^{W}(e) u_{3}\right] \\
\mathcal{M}^{(l, m)}= & -g_{Z W W} P_{W}^{\alpha \beta}\left(p_{1}-p_{2}-k_{1}\right) \Gamma_{\alpha}\left(-k_{1}, p_{1}-p_{2} ; \epsilon_{1}, J_{1}\right) \\
& \times\left[\bar{u}_{4} \gamma_{\beta} g^{W}(e) u_{23, \nu}^{(0)}+\bar{u}_{42, e}^{(m)} \gamma_{\beta} g^{W}(e) u_{3}\right] \\
\mathcal{M}^{(n, o)}= & -g_{Z W W} P_{W}^{\alpha \beta}\left(p_{1}-p_{2}-k_{1}\right) \Gamma_{\alpha}\left(p_{3}-p_{4},-k_{2} ; J_{2}, \epsilon_{2}\right) \\
& \times\left[\bar{u}_{2} \gamma_{\beta} g^{W}(e) u_{11, e}^{(m)}+\bar{u}_{21, \nu}^{(0)} \gamma_{\beta} g^{W}(e) u_{1}\right] \\
\mathcal{M}^{(p-r)}= & \bar{u}_{22, \nu}^{(0)} J_{2} g^{W}(e) u_{11, e}^{(m)}+\bar{u}_{221, \nu \nu}^{(0,0)} J_{2} g^{W}(e) u_{1}+\bar{u}_{2} J_{2} g^{W}(e) u_{211, e e}^{(m, m)} \\
\mathcal{M}^{(s-u)}= & \bar{u}_{42, e}^{(m)} J_{1} g^{W}(e) u_{13, \nu}^{(0)}+\bar{u}_{421, e e}^{(m, m)} J_{1} g^{W}(e) u_{3}+\bar{u}_{4} J_{1} g^{W}(e) u_{213, \nu \nu}^{(0,0)}
\end{aligned}
$$


In the case of $\mathcal{M}^{(f-u)}$ it is understood that similar contributions with $\left(k_{1} \leftrightarrow k_{2}, \epsilon_{1} \leftrightarrow \epsilon_{2}\right)$ are to be added due to identical $Z$ bosons in the final state.

\section{4. $e^{+} e^{-} \rightarrow \nu \bar{\nu} Z Z$ lepton annihilation contributions}

Lepton annihilation contributes via the generic diagrams depicted in Figs. 4(a), (b), (h)-(k), and (p)-(u) with the substitutions

$$
\begin{aligned}
1 & \rightarrow e^{-}, & 2 & \rightarrow e^{-}, \\
3 & \rightarrow \nu, & 4 & \rightarrow \nu, \\
V_{1} & \rightarrow Z, & V_{2} & \rightarrow Z .
\end{aligned}
$$

Here fermion lines 1 and 2 represent the incoming particles. Redefining

$$
\begin{aligned}
& J_{1 \alpha}=\bar{u}_{2} \gamma_{\alpha} g^{Z}(e) u_{1} D^{Z}\left(p_{1}-p_{2}\right), \\
& J_{2 \alpha}=\bar{u}_{4} \gamma_{\alpha} g^{Z}(\nu) u_{3} D^{Z}\left(p_{3}-p_{4}\right),
\end{aligned}
$$

we find the amplitudes

$$
\begin{aligned}
\mathcal{M}^{(a)}= & -\frac{g^{2}}{\left(1-x_{W}\right)^{2}} M_{W}^{2} D^{H}\left(k_{1}+k_{2}\right) J_{1} \cdot J_{2} \epsilon_{1} \cdot \epsilon_{2}, \\
\mathcal{M}^{(b)}= & -\frac{g^{2}}{\left(1-x_{W}\right)^{2}} M_{W}^{2} D^{H}\left(p_{1}-p_{2}-k_{1}\right) \epsilon_{1} \cdot J_{1} \epsilon_{2} \cdot J_{2}, \\
\mathcal{M}^{(h-k)}= & P_{Z}^{\alpha \beta}\left(p_{1}-p_{2}-k_{1}\right)\left[\bar{u}_{2} \gamma_{\alpha} g^{Z}(e) u_{11, e}^{(m)}+\bar{u}_{21, e}^{(m)} \gamma_{\alpha} g^{Z}(e) u_{1}\right] \\
& \times\left[\bar{u}_{4} \gamma_{\beta} g^{Z}(\nu) u_{23, \nu}^{(0)}+\bar{u}_{42, \nu}^{(0)} \gamma_{\beta} g^{Z}(\nu) u_{3}\right], \\
\mathcal{M}^{(p-r)}= & \bar{u}_{22, e}^{(m)} J_{2} g^{Z}(e) u_{11, e}^{(m)}+\bar{u}_{2} J_{2} g^{Z}(e) u_{211, e e}^{(m, m)}+\bar{u}_{221, e e}^{(m, m)} J_{2} g^{Z}(e) u_{1}, \\
\mathcal{M}^{(s-u)}= & \bar{u}_{42, \nu}^{(0)} J_{1} g^{Z}(\nu) u_{13, \nu}^{(0)}+\bar{u}_{4} J_{1} g^{Z}(\nu) u_{213, \nu \nu}^{(0,0)}+\bar{u}_{421, \nu \nu}^{(0,0)} J_{1} g^{Z}(\nu) u_{3} .
\end{aligned}
$$

For the above amplitudes, except for $\mathcal{M}^{(a)}$, similar contributions with $\left(k_{1} \leftrightarrow k_{2}, \epsilon_{1} \leftrightarrow \epsilon_{2}\right)$ have to be added due to identical $Z$ bosons in the final state.

\section{5. $e^{+} e^{-} \rightarrow e^{+} e^{-} W^{+} W^{-}:$Scattering and annihilation contributions}

Both scattering and annihilation contributions arise from the generic diagrams depicted in Figs. 4(a), (c), (f), (g), (i), (k), and (l)-(y) with the substitutions

$$
\begin{aligned}
1 & \rightarrow e^{-}, & 2 & \rightarrow e^{-}, \\
3 & \rightarrow e^{-}, & 4 & \rightarrow e^{-}, \\
V_{1} & \rightarrow W^{-}, & V_{2} & \rightarrow W^{+} .
\end{aligned}
$$

Here fermion lines 1 and 4 ( 1 and 2) are taken to represent the incoming particles in the case of scattering (annihilation). To obtain the full answer, both sets of diagrams have to be added with a relative minus sign between them. Redefining

$$
\begin{aligned}
& J_{1 \alpha}^{V_{1}}=\bar{u}_{2} \gamma_{\alpha} g^{V_{1}}(e) u_{1} D^{V_{1}}\left(p_{1}-p_{2}\right), \\
& J_{2 \alpha}^{V_{2}}=\bar{u}_{4} \gamma_{\alpha} g^{V_{2}}(e) u_{3} D^{V_{2}}\left(p_{3}-p_{4}\right),
\end{aligned}
$$

we find the amplitudes

$$
\begin{aligned}
\mathcal{M}^{(a)}= & -\frac{g^{2}}{1-x_{W}} M_{W}^{2} D^{H}\left(k_{1}+k_{2}\right) \epsilon_{1} \cdot \epsilon_{2} J_{1}^{Z} \cdot J_{2}^{Z} \\
\mathcal{M}^{(c)}= & \sum_{V_{1}, V_{2}=\gamma, Z}-g_{V_{1} W W} g_{V_{2} W W}\left[2 \epsilon_{1} \cdot \epsilon_{2} J_{1}^{V_{1}} \cdot J_{2}^{V_{2}}-\epsilon_{1} \cdot J_{1}^{V_{1}} \epsilon_{2} \cdot J_{2}^{V_{2}}-\epsilon_{1} \cdot J_{2}^{V_{2}} \epsilon_{2} \cdot J_{1}^{V_{1}}\right], \\
\mathcal{M}^{(f)}= & \sum_{V_{1}, V_{2}=\gamma, Z}\left[g_{V_{1} W W} g_{V_{2} W W} P_{W}^{\alpha \beta}\left(p_{1}-p_{2}-k_{1}\right) \Gamma_{\alpha}\left(-k_{1}, p_{1}-p_{2} ; \epsilon_{1}, J_{1}^{V_{1}}\right) \Gamma_{\beta}\left(p_{3}-p_{4},-k_{2} ; J_{2}^{V_{2}}, \epsilon_{2}\right)\right. \\
& \left.\quad+g_{V_{1} W W} g_{V_{2} W W} P_{W}^{\alpha \beta}\left(p_{1}-p_{2}-k_{2}\right) \Gamma_{\alpha}\left(p_{1}-p_{2},-k_{2} ; J_{1}^{V_{1}}, \epsilon_{2}\right) \Gamma_{\beta}\left(-k_{1}, p_{3}-p_{4} ; \epsilon_{1}, J_{2}^{V_{2}}\right)\right],
\end{aligned}
$$




$$
\begin{aligned}
& \mathcal{M}^{(g)}=\sum_{V_{1}, V_{2}=\gamma, Z} g^{2} x_{W} M_{W}^{2} J_{1}^{V_{1}} \cdot \epsilon_{1} J_{2}^{V_{2}} \cdot \epsilon_{2} \frac{\xi}{\xi\left(p_{1}-p_{2}-k_{1}\right)^{2}-M_{W}^{2}} \\
& \times\left\{\begin{array}{ll}
-\tan ^{2} \theta_{W} & \text { if } V_{1}=V_{2}=Z \\
-1 & \text { if } V_{1}=V_{2}=\gamma \\
\tan \theta_{W} & \text { otherwise }
\end{array}\right\} \quad+\left(k_{1} \leftrightarrow k_{2}\right), \\
& \mathcal{M}^{(i)}=P_{W}^{\alpha \beta}\left(p_{1}-p_{2}-k_{1}\right) \bar{u}_{2} \gamma_{\alpha} g^{W}(e) u_{11}^{(0)} \bar{u}_{42}^{(0)} \gamma_{\beta} g^{W}(e) u_{3}, \\
& \mathcal{M}^{(k)}=P_{W}^{\alpha \beta}\left(p_{1}-p_{2}-k_{2}\right) \bar{u}_{22}^{(0)} \gamma_{\alpha} g^{W}(e) u_{1} \bar{u}_{4} \gamma_{\beta} g^{W}(e) u_{13}^{(0)} \text {, } \\
& \mathcal{M}^{(l)}=\sum_{V_{1}=\gamma, Z}-g_{V_{1} W W} P_{W}^{\alpha \beta}\left(p_{1}-p_{2}-k_{1}\right) \Gamma_{\alpha}\left(-k_{1}, p_{1}-p_{2} ; \epsilon_{1}, J_{1}^{V_{1}}\right) \bar{u}_{42}^{(0)} \gamma_{\beta} g^{W}(e) u_{3}, \\
& \mathcal{M}^{(m)}=\sum_{V_{1}=\gamma, Z}-g_{V_{1} W W} P_{W}^{\alpha \beta}\left(p_{1}-p_{2}-k_{2}\right) \Gamma_{\alpha}\left(p_{1}-p_{2},-k_{2} ; J_{1}^{V_{1}}, \epsilon_{2}\right) \bar{u}_{4} \gamma_{\beta} g^{W}(e) u_{13}^{(0)}, \\
& \mathcal{M}^{(n)}=\sum_{V_{2}=\gamma, Z}-g_{V_{2} W W} P_{W}^{\alpha \beta}\left(p_{1}-p_{2}-k_{2}\right) \Gamma_{\alpha}\left(-k_{1}, p_{3}-p_{4} ; \epsilon_{1}, J_{2}^{V_{2}}\right) \bar{u}_{22}^{(0)} \gamma_{\beta} g^{W}(e) u_{1}, \\
& \mathcal{M}^{(o)}=\sum_{V_{2}=\gamma, Z}-g_{V_{2} W W} P_{W}^{\alpha \beta}\left(p_{1}-p_{2}-k_{1}\right) \Gamma_{\alpha}\left(p_{3}-p_{4},-k_{2} ; J_{2}^{V_{2}}, \epsilon_{2}\right) \bar{u}_{2} \gamma_{\beta} g^{W}(e) u_{11}^{(0)}, \\
& \mathcal{M}^{(p-r)}=\sum_{V_{2}=\gamma, Z}\left[\bar{u}_{22}^{(0)} J_{2}^{V_{2}} g^{V_{2}}(\nu) u_{11}^{(0)}+\bar{u}_{2} J_{2}^{V_{2}} g^{V_{2}}(e) u_{211}^{(m, 0)}+\bar{u}_{221}^{(0, m)} J_{2}^{V_{2}} g^{V_{2}}(e) u_{1}\right], \\
& \mathcal{M}^{(s-u)}=\sum_{V_{1}=\gamma, Z}\left[\bar{u}_{42}^{(0)} J_{1}^{V_{1}} g^{V_{1}}(\nu) u_{13}^{(0)}+\bar{u}_{4} J_{1}^{V_{1}} g^{V_{1}}(e) u_{213}^{(m, 0)}+\bar{u}_{421}^{(0, m)} J_{1}^{V_{1}} g^{V_{1}}(e) u_{3}\right] \\
& \mathcal{M}^{(v)}=\sum_{V_{1}, V_{2}=\gamma, Z}-g_{V_{2} W W} D^{V_{2}}\left(k_{1}+k_{2}\right) \bar{u}_{4} \not{W}\left(k_{1}, k_{2} ; \epsilon_{1}, \epsilon_{2}\right) g^{V_{2}}(e) \frac{\not p_{4}+\not k_{1}+\not k_{2}+m}{\left(p_{4}+k_{1}+k_{2}\right)^{2}-m^{2}} J_{1}^{V_{1}} g^{V_{1}}(e) u_{3}, \\
& \mathcal{M}^{(w)}=\sum_{V_{1}, V_{2}=\gamma, Z}-g_{V_{2} W W} D^{V_{2}}\left(k_{1}+k_{2}\right) \bar{u}_{4} J_{1}^{V_{1}} g^{V_{1}}(e) \frac{\not p_{3}-\not k_{1}-\not k_{2}+m}{\left(p_{3}-k_{1}-k_{2}\right)^{2}-m^{2}} \mp\left(k_{1}, k_{2} ; \epsilon_{1}, \epsilon_{2}\right) g^{V_{2}}(e) u_{3}, \\
& \mathcal{M}^{(x)}=\sum_{V_{1}, V_{2}=\gamma, Z}-g_{V_{1} W W} D^{V_{1}}\left(k_{1}+k_{2}\right) \bar{u}_{2} J_{2}^{V_{2}} g^{V_{2}}(e) \frac{\not p_{1}-\not k_{1}-\not k_{2}+m}{\left(p_{1}-k_{1}-k_{2}\right)^{2}-m^{2}} \not\left(k_{1}, k_{2} ; \epsilon_{1}, \epsilon_{2}\right) g^{V_{1}}(e) u_{1}, \\
& \mathcal{M}^{(y)}=\sum_{V_{1}, V_{2}=\gamma, Z}-g_{V_{1} W W} D^{V_{1}}\left(k_{1}+k_{2}\right) \bar{u}_{2} \not\left(k_{1}, k_{2} ; \epsilon_{1}, \epsilon_{2}\right) g^{V_{1}}(e) \frac{\not p_{2}+\not k_{1}+\not k_{2}+m}{\left(p_{2}+k_{1}+k_{2}\right)^{2}-m^{2}} J_{2}^{V_{2}} g^{V_{2}}(e) u_{1} \text {. }
\end{aligned}
$$

\section{6. $e^{+} e^{-} \rightarrow e^{+} e^{-} Z Z$ : Scattering and annihilation contributions}

Both scattering and annihilation contributions arise from the generic diagrams depicted in Figs. 4(a), (b), (h)-(k), and $(\mathrm{p})-(\mathrm{u})$ with the substitutions

$$
\begin{aligned}
1 & \rightarrow e^{-}, & 2 & \rightarrow e^{-}, \\
3 & \rightarrow e^{-}, & 4 & \rightarrow e^{-}, \\
V_{1} & \rightarrow Z, & V_{2} & \rightarrow Z .
\end{aligned}
$$

Here fermion lines 1 and 4 ( 1 and 2 ) are taken to represent the incoming particles in the case of scattering (annihilation). To obtain the full answer, both sets of diagrams have to be added with a relative minus sign between them. Redefining

$$
\begin{aligned}
& J_{1 \alpha}^{V}=\bar{u}_{2} \gamma_{\alpha} g^{V}(e) u_{1} D^{V}\left(p_{1}-p_{2}\right), \\
& J_{2 \alpha}^{V}=\bar{u}_{4} \gamma_{\alpha} g^{V}(e) u_{3} D^{V}\left(p_{3}-p_{4}\right),
\end{aligned}
$$

we find the amplitudes 


$$
\begin{aligned}
\mathcal{M}^{(a)} & =-\frac{g^{2}}{\left(1-x_{W}\right)^{2}} M_{W}^{2} D^{H}\left(k_{1}+k_{2}\right) J_{1}^{Z} \cdot J_{2}^{Z} \epsilon_{1} \cdot \epsilon_{2} \\
\mathcal{M}^{(b)} & =-\frac{g^{2}}{\left(1-x_{W}\right)^{2}} M_{W}^{2} D^{H}\left(p_{1}-p_{2}-k_{1}\right) \epsilon_{1} \cdot J_{1}^{Z} \epsilon_{2} \cdot J_{2}^{Z} \\
\mathcal{M}^{(h-k)} & =\sum_{V=\gamma, Z} P_{V}^{\alpha \beta}\left(p_{1}-p_{2}-k_{1}\right)\left[\bar{u}_{2} \gamma_{\alpha} g^{V}(e) u_{11, e}^{(m)}+\bar{u}_{21, e}^{(m)} \gamma_{\alpha} g^{V}(e) u_{1}\right] \\
\mathcal{M}^{(p-r)} & =\sum_{V=\gamma, Z}\left[\bar{u}_{22, e}^{(m)} \gamma_{2}^{V} g^{V}(e) u_{11, e}^{(m)}+\bar{u}_{2} J_{2}^{V} g^{V}(e) u_{211, e e}^{(m, m)}+\bar{u}_{221, e e}^{(m, m)} J_{2}^{V} g^{V}(e) u_{1}\right] \\
\mathcal{M}^{(s-u)} & =\sum_{V=\gamma, Z}\left[\bar{u}_{42, e}^{(m)} J_{1}^{V} g^{V}(e) u_{13, e}^{(m)}+\bar{u}_{4} J_{1}^{V} g^{V}(e) u_{213, e e}^{(m, m)}+\bar{u}_{421, e e}^{(m, m)} J_{1}^{V} g^{V}(e) u_{3}\right]
\end{aligned}
$$

For the above amplitudes, except for $\mathcal{M}^{(a)}$, similar contributions with $\left(k_{1} \leftrightarrow k_{2}, \epsilon_{1} \leftrightarrow \epsilon_{2}\right)$ have to be added due to identical $Z$ bosons in the final state.

\section{7. $e^{+} e^{-} \rightarrow e^{+} \nu W^{-} Z$ : Scattering and annihilation channels}

Both scattering and annihilation contributions arise from the generic diagrams depicted in Figs. 4(b)-(r), (t), (u), and $(\mathrm{w})-(\mathrm{y})$ with the substitutions

$$
\begin{aligned}
1 & \rightarrow e^{-}, & 2 & \rightarrow \nu, \\
3 & \rightarrow e^{-}, & 4 & \rightarrow e^{-}, \\
V_{1} & \rightarrow W^{-}, & V_{2} & \rightarrow Z .
\end{aligned}
$$

Here fermion lines 1 and 4 ( 3 and 4 ) are taken to represent the incoming particles in the case of scattering (annihilation). To obtain the full answer, both sets of diagrams have to be added with a relative minus sign between them. Redefining

$$
\begin{gathered}
J_{1 \alpha}=\bar{u}_{2} \gamma_{\alpha} g^{W}(e) u_{1} D^{W}\left(p_{1}-p_{2}\right), \\
J_{2 \alpha}^{V}=\bar{u}_{4} \gamma_{\alpha} g^{V}(e) u_{3} D^{V}\left(p_{3}-p_{4}\right),
\end{gathered}
$$

we find for the amplitudes

$$
\begin{aligned}
& \mathcal{M}^{(b)}=-\frac{g^{2}}{1-x_{W}} M_{W}^{2} D^{H}\left(p_{1}-p_{2}-k_{1}\right) \epsilon_{1} \cdot J_{1} \epsilon_{2} \cdot J_{2}^{Z} \\
& \mathcal{M}^{(c)}=\sum_{V=\gamma, Z}-g_{V W W} g_{Z W W}\left[2 \epsilon_{1} \cdot J_{1} \epsilon_{2} \cdot J_{2}^{V}-\epsilon_{1} \cdot J_{2}^{V} \epsilon_{2} \cdot J_{1}-\epsilon_{1} \cdot \epsilon_{2} J_{1} \cdot J_{2}^{V}\right] \\
& \mathcal{M}^{(d)}=\sum_{V=\gamma, Z} g_{V W W} g_{Z W W} P_{W}^{\alpha \beta}\left(k_{1}+k_{2}\right) \Gamma_{\alpha}\left(k_{2}, k_{1} ; \epsilon_{2}, \epsilon_{1}\right) \Gamma_{\beta}\left(p_{3}-p_{4}, p_{1}-p_{2} ; J_{2}^{V}, J_{1}\right) \\
& \mathcal{M}^{(e)}=\sum_{V=\gamma, Z} g^{2} x_{W} M_{W}^{2} \epsilon_{1} \cdot \epsilon_{2} J_{1} \cdot J_{2}^{V} \frac{\xi}{\xi\left(k_{1}+k_{2}\right)^{2}-M_{W}^{2}} \\
& \times\left\{\begin{array}{cl}
-\tan ^{2} \theta_{W} & \text { if } V=Z, \\
\tan \theta_{W} & \text { if } V=\gamma,
\end{array}\right. \\
& \mathcal{M}^{(f)}=\sum_{V=\gamma, Z} g_{V W W} g_{Z W W} P_{W}^{\alpha \beta}\left(p_{1}-p_{2}-k_{2}\right) \Gamma_{\alpha}\left(-k_{2}, p_{1}-p_{2} ; \epsilon_{2}, J_{1}\right) \Gamma_{\beta}\left(-k_{1}, p_{3}-p_{4} ; \epsilon_{1}, J_{2}^{V}\right) \\
& \mathcal{M}^{(g)}=\sum_{V=\gamma, Z} g^{2} x_{W} M_{W}^{2} \epsilon_{1} \cdot J_{2}^{V} \epsilon_{2} \cdot J_{1} \frac{\xi}{\xi\left(p_{1}-p_{2}-k_{2}\right)^{2}-M_{W}^{2}} \\
& \times\left\{\begin{array}{cl}
-\tan ^{2} \theta_{W} & \text { if } V=Z, \\
\tan \theta_{W} & \text { if } V=\gamma,
\end{array}\right. \\
& \mathcal{M}^{(h-k)}=P_{Z}^{\alpha \beta}\left(p_{1}-p_{2}-k_{1}\right) \bar{u}_{2} \gamma_{\alpha} g^{Z}(\nu) u_{11}^{(0)}\left[\bar{u}_{42, e}^{(m)} \gamma_{\beta} g^{Z}(e) u_{3}+\bar{u}_{4} \gamma_{\beta} g^{Z}(e) u_{23, e}^{(m)}\right] \\
& +\sum_{V=\gamma, Z} P_{V}^{\alpha \beta}\left(p_{1}-p_{2}-k_{1}\right) \bar{u}_{21}^{(m)} \gamma_{\alpha} g^{V}(e) u_{1}\left[\bar{u}_{4} \gamma_{\beta} g^{V}(e) u_{23, e}^{(m)}+\bar{u}_{42, e}^{(m)} \gamma_{\beta} g^{V}(e) u_{3}\right] \\
& +P_{W}^{\alpha \beta}\left(p_{1}-p_{2}-k_{2}\right) \bar{u}_{4} \gamma_{\alpha} g^{W}(e) u_{13}^{(0)}\left[\bar{u}_{2} \gamma_{\beta} g^{W}(e) u_{21, e}^{(m)}+\bar{u}_{22, \nu}^{(0)} \gamma_{\beta} g^{W}(e) u_{1}\right] \text {, }
\end{aligned}
$$




$$
\begin{aligned}
\mathcal{M}^{(l-o)}= & \sum_{V=\gamma, Z}-g_{V W W} P_{V}^{\alpha \beta}\left(p_{1}-p_{2}-k_{1}\right) \Gamma_{\alpha}\left(p_{1}-p_{2},-k_{1} ; J_{1}, \epsilon_{1}\right) \\
& \times\left[\bar{u}_{42, e}^{(m)} \gamma_{\beta} g^{V}(e) u_{3}+\bar{u}_{4} \gamma_{\beta} g^{V}(e) u_{23, e}^{(m)}\right] \\
& -g_{Z W W} P_{W}^{\alpha \beta}\left(p_{1}-p_{2}-k_{2}\right) \Gamma_{\alpha}\left(-k_{2}, p_{1}-p_{2} ; \epsilon_{2}, J_{1}\right) \bar{u}_{4} \gamma_{\beta} g^{W}(e) u_{13}^{(0)} \\
& +\sum_{V=\gamma, Z}-g_{V W W} P_{W}^{\alpha \beta}\left(p_{1}-p_{2}-k_{2}\right) \Gamma_{\alpha}\left(-k_{1}, p_{3}-p_{4} ; \epsilon_{1}, J_{2}^{V}\right) \\
& \times\left[\bar{u}_{22, \nu}^{(0)} \gamma_{\beta} g^{W}(e) u_{1}+\bar{u}_{2} \gamma_{\beta} g^{W}(e) u_{21, e}^{(m)}\right] \\
\mathcal{M}^{(p-r)}= & \sum_{V=\gamma, Z}\left[\bar{u}_{21}^{(m)} j_{2}^{V} g^{V}(e) u_{21, e}^{(m)}+\bar{u}_{212, e}^{(m, m)} j_{2}^{V} g^{V}(e) u_{1}+\bar{u}_{221, \nu}^{(0, m)} j_{2}^{V} g^{V}(e) u_{1}\right] \\
& +\bar{u}_{2} J_{2}^{Z} g^{Z}(\nu) u_{211, \nu}^{(0,0)}+\bar{u}_{22, \nu}^{(0)} J_{2}^{Z} g^{Z}(\nu) u_{11}^{(0)}+\bar{u}_{2} J_{2}^{Z} g^{Z}(\nu) u_{121, e}^{(0, m)} \\
\mathcal{M}^{(t, u)}= & \bar{u}_{42, e}^{(m)} J_{1} g^{W}(e) u_{13}^{(0)}+\bar{u}_{4} J_{1} g^{W}(e) u_{213, \nu}^{(0,0)}+\bar{u}_{2} J_{1} g^{W}(e) u_{123, e}^{(0, m)} \\
\mathcal{M}^{(w)}= & -g_{Z W W} P_{W}^{\alpha \beta}\left(k_{1}+k_{2}\right) \Gamma_{\alpha}\left(k_{2}, k_{1} ; \epsilon_{2}, \epsilon_{1}\right) \bar{u}_{4} J_{1} g^{W}(e) \frac{\not p_{3}-\not k_{1}-\not k_{2}}{\left(p_{3}-k_{1}-k_{2}\right)^{2} \gamma_{\beta} g^{W}(e) u_{3}} \\
\mathcal{M}^{(x)}= & -g_{Z W W} P_{W}^{\alpha \beta}\left(k_{1}+k_{2}\right) \Gamma_{\alpha}\left(k_{2}, k_{1} ; \epsilon_{2}, \epsilon_{1}\right) \bar{u}_{2} J_{2}^{Z} g^{Z}(\nu) \frac{\not p_{1}-\not k_{1}-\not k_{2}}{\left(p_{1}-k_{1}-k_{2}\right)^{2} \gamma_{\beta} g^{W}(e) u_{1}} \\
\mathcal{M}^{(y)}= & \sum_{V=\gamma, Z}-g_{Z W W} P_{W}^{\alpha \beta}\left(k_{1}+k_{2}\right) \Gamma_{\alpha}\left(k_{2}, k_{1} ; \epsilon_{2}, \epsilon_{1}\right) \bar{u}_{2} \gamma_{\beta} g^{W}(e) \frac{\not p_{2}+\not k_{1}+\not k_{2}+m}{\left(p_{2}+k_{1}+k_{2}\right)^{2}-m^{2}} J_{2}^{V} g^{V}(e) u_{1} .
\end{aligned}
$$

The matrix elements for $e^{+} e^{-} \rightarrow \bar{\nu} e^{-} W^{+} Z$ can be obtained from those above by $C P$ conjugation.

[1] See, for reference, Research Directions for the Decade, Proceedings of the Snowmass Summer Study on High Energy Physics; Snowmass, Colorado, 1990, edited by E. L. Berger and I. Butler (World Scientific, Singapore, 1991); Proceedings of the ECFA Large Hadron Collider Workshop, Aachen, Germany, 1990, edited by G. Jarlskog and D. Rein (CERN Report No. CERN 90-10, Geneva, Switzerland, 1990), Vol. II, p. 427.

[2] See, for reference, Proceedings of the Workshop on Physics at Future Accelerators, La Thuile, Italy, 1987, edited by J. H. Mulvey (CERN Yellow Report No. 87-07, Geneva, Switzerland, 1987), Vols. I and II; C. Ahn et al., SLAC Report No. 329, 1988 (unpublished); Proceedings of the First Workshop on Japan Linear Collider (JLC), Tsukuba, Japan, 1989, edited by S. Kawabata (KEK Report No. 90-2, Tsukuba, 1990); Proceedings of the 2nd International Workshop on Next-Generation Linear Colliders: LC90, Tsukuba, Japan, 1990, edited by S. Kurokawa et al. (KEK Internal Report No. 90-22, Tsukuba, 1990).

[3] K. Hagiwara, J. Kanzaki, and H. Murayama, KEK Report No. 91-4 (unpublished); H. Murayama, Ph.D. thesis, University of Tokyo Report No. UT-580, 1991.

[4] J. F. Gunion, P. Kalyniak, M. Soldate, and P. Galison, Phys. Rev. D 34, 101 (1986).

[5] B. W. Lee, C. Quigg, and H. B. Thacker, Phys. Rev. D 16, 1519 (1977); R. N. Cahn, Nucl. Phys. B255, 341 (1985).

[6] V. Barger, in Proceedings of the XXIVth International Conference on High Energy Physics, Munich, West Germany, 1988, edited by R. Kotthaus and J. Kuhn (Springer-Verlag, Heidelberg, 1989), p. 1265.
[7] Z. Kunszt and W. J. Stirling, Phys. Lett. B 242, 507 (1990); V. Barger and K. Whisnant, Phys. Rev. D 42, 138 (1990); J. F. Gunion and L. Roszkowski, University of California-Davis Report No. UCD-90-26 (unpublished); N. Brown, Z. Phys. C 49, 657 (1991).

[8] V. Barger, G. Bhattacharya, T. Han, and B. A. Kniehl, Phys. Rev. D 43, 779 (1991).

[9] B. A. Kniehl, Nucl. Phys. B376, 3 (1992).

[10] S. G. Gorishny, A. L. Kataev, S. A. Larin, and L. R. Surgaladze, Mod. Phys. Lett. A 5, 2703 (1990).

[11] J. Gasser and H. Leutwyler, Phys. Rep. 87, 77 (1982); H. Leutwyler, Nucl. Phys. B337, 108 (1990); Z. Kunszt and W. J. Stirling, in Proceedings of the ECFA Large Hadron Collider Workshop [1], Vol. II, p. 428.

[12] S. Dawson, Nucl. Phys. B359, 283 (1991); A. Djouadi, M. Spira, and P. M. Zerwas, Phys. Lett. B 264, 440 (1991).

[13] LEP Collaborations: ALEPH, DELPHI, L3, and OPAL, Phys. Lett. B 276, 247 (1992).

[14] A. Tofighi-Niaki and J. F. Gunion, Phys. Rev. D 36, 2671 (1987); 38, 1433 (1988).

[15] V. Barger, J. Hewett, and T. Rizzo, Phys. Rev. Lett. 65, 1313 (1990); F. Halzen and D. Morris, Phys. Lett. B 237, 107 (1990); F. Halzen, B. A. Kniehl, and M. L. Stong, UW-Madison Report No. MAD/PH/658, 1991 (unpublished); J. Ellis and G. Fogli, Phys. Lett. B 249, 543 (1990); J. Ellis, G. Fogli, and E. Lisi, ibid. 274, 456 (1992).

[16] CDF Collaboration, F. Abe et al., Phys. Rev. Lett. 68, 447 (1992).

[17] V. N. Baier and V. M. Katkov, Phys. Lett. 25A, 492 
(1967).

[18] P. R. Burchat, D. L. Burke, and A. Petersen, Phys. Rev. D 38, 2735 (1992).

[19] V. Barger, A. Stange, and R. J. N. Phillips, Phys. Rev. D 45, 1484 (1992).

[20] U. Baur and E. W. N. Glover, Phys. Lett. B 252, 683 (1990).

[21] V. Barger et al., Phys. Rev. D 30, 947 (1984); I. Bigi et al., Phys. Lett. B 181, 157 (1986); V. Barger, J. Ohnemus, and R. J. N. Phillips, Int. J. Mod. Phys. A 4, 617 (1989).

[22] C. Peterson et al., Phys. Rev. D 27, 105 (1983).

[23] L3 collaboration, B. Adeva et al., Phys. Lett. B 261, 177 (1991).

[24] B. A. Kniehl, Z. Phys. C 55, 605 (1992).

[25] V. V. Sudakov, Zh. Eksp. Teor. Fiz. 30, 87 (1956) [Sov. Phys. JETP 3, 65 (1956)].

[26] T. Kinoshita, J. Math. Phys. 3, 650 (1962); T. D. Lee and M. Nauenberg, Phys. Rev. 133, B1549 (1964).

[27] D. R. Yennie, S. C. Frautschi, and H. Suura, Ann. Phys. (N.Y.) 13, 379 (1961); G. Grammer, Jr. and D. R. Yennie, Phys. Rev. D 8, 4332 (1973).

[28] F. A. Berends, G. J. H. Burgers, and W. L. van Neerven, Nucl. Phys. B297, 429 (1988); B304, 921(E) (1988);
B. A. Kniehl, M. Krawczyk, J. H. Kühn, and R. G. Stuart, Phys. Lett. B 209, 337 (1988); B. A. Kniehl, ibid. 237, 127 (1990).

[29] E. A. Kuraev and V. S. Fadin, Yad. Fiz. 41, 733 (1985) [Sov. J. Nucl. Phys. 41, 466 (1985)]; G. Altarelli and G. Martinelli, in Physics at LEP, LEP Jamboree, Geneva, Switzerland, 1985, edited by J. Ellis and R. Peccei (CERN Yellow Report No. 86-02, Geneva, 1986), Vol. 1, p. 47; O. Nicrosini and L. Trentadue, Phys. Lett. B 196, 551 (1987).

[30] T. Barklow (private communication).

[31] R. B. Palmer, Annu. Rev. Nucl. Part. Sci. 40, 529 (1990).

[32] P. Igo-Kemenes et al., in Proceedings of the Workshop on Physics and Experiments with Linear Colliders, Saariselkä, Finland, 1991, edited by R. Orava, P. Eerola, and M. Nordberg (World Scientific, Singapore, in press).

[33] G.-A. Voss, talk given at Ringberg Workshop: High Precision versus High Energies in $e^{+} e^{-}$Collisions, Tegernsee, Germany, 1991 (unpublished).

[34] T. Weiland, in Proceedings of the Workshop on Physics and Experiments With Linear Colliders [32].

[35] B. Wiik, talk given at Munich, Germany (unpublished).

[36] R. J. Noble, Nucl. Instrum. Methods A256, 427 (1987). 